\title{
Experimental and numerical study of separation characteristics in gas-liquid cylindrical cyclone
}

\author{
Lele Yang ${ }^{\mathrm{a}, \mathrm{c}}$, Jan Zhang ${ }^{\mathrm{b}}$, Yong Ma ${ }^{\mathrm{a}, \mathrm{c}, *}$, Jingyu Xu ${ }^{\mathrm{b}, *}$, Jing Wang ${ }^{\mathrm{a}}$ \\ ${ }^{a}$ School of Marine Engineering and Technology, Sun Yat-sen University, Guangzhou 518000, China \\ ${ }^{\mathrm{b}}$ Institute of Mechanics, Chinese Academy of Sciences, Beijing 100190, China \\ ' Southern Marine Science and Engineering Guangdong Laboratory (Zhuhai), Zhuhai 519000, China
}

\section{H I G H L I G H T S}

- The breakup, coalescence, and migration regularity of droplets are focused on.

- The existence of the flow reversal for the gas core is proved by ERT measurement results.

- The numerical model considering droplets breakup and coalescence is developed.

- The developed droplet migration model can accurately predict the separation performance of GLCC.

\section{A R T I C L E I N F O}

\section{Article history:}

Received 19 August 2019

Received in revised form 21 October 2019

Accepted 16 November 2019

Available online 18 November 2019

\section{Keywords:}

Gas-liquid cylinder cyclone

Droplets

Breakup

Coalescence

Migration model

\begin{abstract}
A B S T R A C T
The separation characteristics of the gas-liquid cylindrical cyclone (GLCC) has been investigated by experiment and numerical analysis. The droplet size and phase distributions were measured using Malvern RTsizer and electrical resistivity tomography, respectively. The Discrete Phase Model was used to numerically analyze the swirling hydrodynamics. The results showed that the separator with strong swirl intensity would not necessarily get better separation performance, and the nozzle with $\mathrm{Ne}=5.9$ performed best. The small and medium droplets tended to initially coalesce and subsequently break up with increased gas superficial velocity, whereas they always tended to coalesce with increased liquid superficial velocity. ERT measurement results proved the existence of the flow reversal for the gas core. Finally, a droplet migration model was developed based on the force analysis of droplet and the swirling hydrodynamics, which accurately predicted the separation performance of GLCC. These results can be used in the design of the GLCC.
\end{abstract}

() 2019 Elsevier Ltd. All rights reserved.

\section{Introduction}

Traditionally, the oil industry has relied on conventional vesseltype separators to separate natural gas from the gas-liquid mixtures produced from oil/gas wells. These separators rely on gravity and expansion, and are bulky, heavy, and costly. With the rise in offshore exploitations, compact alternatives are desired to cut down the costs associated with the separation equipment. The gas-liquid cylindrical cyclone (GLCC), which mainly involves the combination of gravitational and centrifugal forces to realize separation, may be the solution. The GLCC exhibits high performance

\footnotetext{
* Corresponding authors at: School of Marine Engineering and Technology, Sun Yat-sen University, Guangzhou 518000, China (Y. Ma); Institute of Mechanics, Chinese Academy of Sciences, Beijing 100190, China (J. Xu).

E-mail addresses: mayong3@mail.sysu.edu.cn (Y. Ma), xujingyu@imech.ac.cn
} (J. Xu). and has a relatively small size owing to its short fluid residence durations.

The GLCC has been used in the fields where partial or complete gas-liquid separation is required. It can be operated as a multiphase metering loop, where gas and liquid are separately metered by gas and liquid flow meters. With the gas and liquid outlets recombined, the metering loop has some self-regulating functions that can reduce or even eliminate the need for liquid-level control (Wang et al., 2010). The GLCC is better suited to applications requiring only partial gas-liquid separation. The raw gas, separated by the GLCC from high-pressure wells, can be used for the gas lift of low-pressure wells (Kouba and Shoham, 1996). Moreover, fluctuations in the liquid flow are reduced, which can improve the performance of downstream equipment, such as multiphase pump, desander, and oil-water cyclone separator (Arpandi et al., 1996).

Despite decades of research, it is still difficult to predict the complex hydrodynamic performance of the GLCC. Thus, it is 
difficult to reasonably determine the structural dimension according to the condition of the produced liquid, which has been the main obstruction to extending the application of the GLCC. Today, the research on the GLCC are mainly based on the "black box" theory, which involves observing the change in separation performance with structure parameters and operation parameters. Kouba et al. (1995) observed that inclining the inlet approximately $27^{\circ}$ downward significantly reduced the amount of liquid carryover. Movafaghian et al. (2000) studied the effects of geometry, fluid properties, and pressure on the separation performance of the GLCC. Wang (1997) found that the applicable operating conditions can be extended using an inlet nozzle with a reduced size. Meléndez-Ramírez et al. (2004) presented that the separator efficiency was mainly affected by the inlet gas flow rate for the explored conditions with slug flow at the GLCC entrance. However, these previous studies seldom mentioned the relationship between the internal flow field and separation performance.

In fact, fewer experimental studies investigate the internal flow field in the GLCC. Erdal and Shirazi (2004) measured the axial and tangential velocities of the single phase swirling flow at 24 different axial locations in the range from a distance of $32 \mathrm{~cm}$ to $90 \mathrm{~cm}$ below the inlet by using a Laser Doppler Velocimeter (LDV). Similarly using the LDV to measure the velocity field in the lower part of the GLCC, Hreiz et al. (2014) gave an understanding of the origin of the double flow reversal regime that was encountered in swirl flows. In the upper part of the GLCC, the liquid phase generally consists of both liquid film and droplets that can transform into each other and fall back under the effect of gravity and centrifugal force. Due to this complex hydrodynamics behavior of gas-liquid swirl flow, it is difficult to investigate the velocity distribution in the upper part of the GLCC by experimental measurement.

Numerical simulation methods can provide detailed and quantitative velocity distribution in the the GLCC. Hreiz et al. (2011) calculated the single-phase hydrodynamics for the swirling flow in the GLCC by employing several turbulence RANS and LES models. Through Eulerian multiphase model of ANSYS Fluent, Yue et al (2019) analysed the flow behvior of liquid film based on the gas tangential and axial velocities in the upper part of the GLCC. Using CFX, Reyes-Gutiérrez et al. (2006) simulated single-phase and twophase flows in the GLCC, and the simulated interface vortex shape and liquid angular velocity showed a reasonable match with experimental data. However, most of these previous studies on CFD simulation of GLCC separator are restricted to single phase flow or ignore the effect of droplet breakup and coalescence on the gas flow field.

In fact, liquid carry-over and gas carry-under hinder the separation efficiency of the GLCC. Liquid carry-over and gas carry-under refer to the entrainment of liquid into an exiting gas stream and the entrainment of gas into an exiting liquid stream, respectively. In terms of the liquid carry-over, the droplet size distributions are essential because it directly affects the centrifugal effect of the droplets. Breakup and coalescence regularity of non-dilute oil drops in a vane-type swirling flow field has been investigated according to the drop size distribution measured by Malvern RTsizer (Liu et al., 2018). The research on the droplet size distribution in the GLCC has not yet found in the literatures. And for gas carryunder, the geometry and the stability of the gas core have a strong influence (Bergström and Vomhoff, 2007). To avoid interference in the swirling flow, nonintrusive measurement mehods were required for direct measurement of the gas core. Williams et al. (1995) successfully used electrical resistance tomography (ERT) to monitor the stability of the air-core in the cyclonic sepators. In order to determine the initiation of gas carry-under, many mechanistic models have been developed and improved (Mantilla, 1998; Kouba et al., 1995). However, there have been no mechanistic models and fundamental work published to reasonably predict the liquid carry-over.

In this work, the droplet size distribution in the GLCC was firstly measured experimentally using a Malvern RTsizer, and the gas core in the lower part of the GLCC was monitored by ERT system. Considering the effect of droplet breakup and coalescence on the gas flow field, the hydrodynamics in the GLCC was numerically simulated using Discrete Phase Model (DPM) coupled with RNG $k-\varepsilon$ turbulence model. Furthermore, based on the analysis of the forces acting on the droplet and the distribution characteristics of the swirl flow field, a droplet migration model was developed to predict the liquid carry-over in the GLCC. Through the mechanistic model, the relationship between the characteristics of the swirling flow field and separation efficiency was established.

\section{Experimental investigation}

\subsection{Test configuration}

The GLCC body was fabricated from plexiglass to enable visual observation. Fig. 1 shows the schematic of the GLCC. The GLCC has a $50 \mathrm{~mm}$ I.D. pipe mounted vertically with a total height of $1980 \mathrm{~mm}$. The gas leg is a $25 \mathrm{~mm}$ I.D. pipe located $1070 \mathrm{~mm}$ from the inlet. The liquid leg is a $50 \mathrm{~mm}$ I.D. pipe located $780 \mathrm{~mm}$ from the inlet.

The inlet is $25 \mathrm{~mm}$ I.D. pipe inclined downwards to cause the swirling liquid to run below the inlet, thereby allowing the unobstructed passage of the gas into the upper part of the GLCC. In this study, a downward inclination angle of $27^{\circ}$ was selected to retard the onset of the liquid carry-over, which has been generally recommended by many scholars (Arpandi et al., 1996; Chirinos et al., 1999; Kouba et al., 1995; Mohan et al., 1998). The necking ratio is an important parameter for obtaining the swirl intensity and it is defined as:

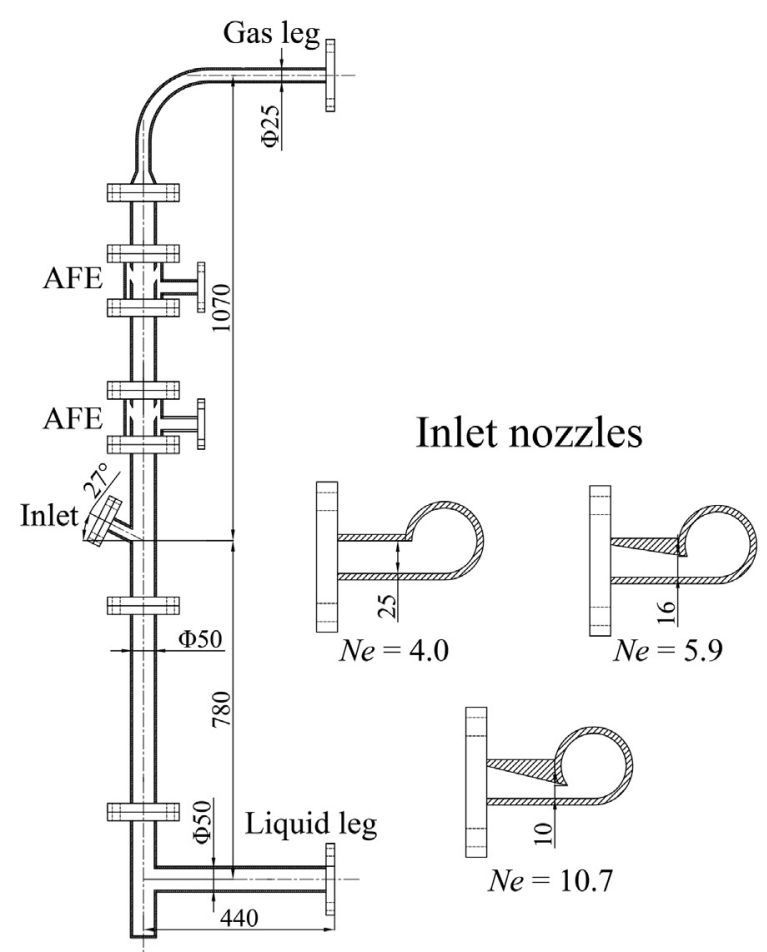

Fig. 1. Schematic of the GLCC and three types of inlet nozzles. 
$\mathrm{Ne}=\frac{A_{G L C C}}{A_{\text {slot }}}$.

where $A_{G L C C}$ and $A_{\text {slot }}$ are the cross-sectional area of the GLCC body and the slot at the inlet, respectively.

To investigate the effect of the necking ratio on the separation performance, three types of inlet structures were used: $\mathrm{Ne}=4.0$, 5.9, and 10.7. All the inlets were tangents to the body, as shown in Fig. 1. The data in the following image, unless specified, corresponds to the nozzle with $\mathrm{Ne}=4.0$.

Two annular film extractors (AFEs) were applied to measure the droplet size distribution and improve the separation performance of the GLCC. The AFEs consisted of annular tubes and a liquid leg (I.D. of $25 \mathrm{~mm}$ ). The diameters of the inner tube and outer tube inlet were $50 \mathrm{~mm}$ and $80 \mathrm{~mm}$, respectively. The liquid film could be removed from the annular gap of the inner tube and could flow out through the liquid leg. The low AFEs and the high AFEs were located $240 \mathrm{~mm}$ and $555 \mathrm{~mm}$ above the inlet centerline, respectively.

\subsection{Measuring method and systematic error}

Based on the phase Doppler particle analyzer (PDPA), Santolaya et al. (2013) and Sommerfeld (1998) measured the droplet size distribution of sprays generated by pressure swirl atomizers, although the signals from multiple and non-spherical droplets could not be well received by the PDPA. By analyzing the droplet images recorded by the high-speed camera, the probability density function of the droplet size distribution of the spray or liquid entrainment in dispersed flow was obtained (Lan et al., 2014; Patruno et al., 2010). Other measuring techniques such as sieving, resistance method, and microscope observation were not suitable for measuring the wide droplet size distribution. The Malvern particle sizer is a powerful tool for time-averaged and fast online measurements of the droplet size distribution. Ding et al. (2017) measured the droplet size of a coaxial spray using a Malvern RTsizer. Simmons and Hanratty (2001) investigated the droplet size distribution in a horizontal annular gas-liquid flow using a Malvern Spraytec R5008 particle sizer.

Malvern instruments are based on laser diffraction techniques. Light scattering phenomenon occurs when a laser beam passes through a group of droplets. The scattered light is focused using a Fourier lens, after which it is detected using an annular detector array. Thereafter, the scattered light is converted into a far-field diffraction pattern. The droplet size can be determined by the diffraction angle since the diffraction angle varies inversely to the droplet size. For the far-field diffraction, the energy distribution of the scattered light is only related to the droplet size distribution. Thus, the droplet size distribution can be reversecalculated according to the energy spectrum. However, it is difficult to evaluate the error of the Malvern instrument because all the available methods for particle size distribution suffer from inherent flaws (Eshel et al., 2004). Kusters et al. (1991) found that serious errors could occur when a Malvern 2600 instrument using a scattering matrix corresponding to a refractive-index ratio of about 1.2 is applied to measure systems with refractive-index ratios near 1. In this study, the refractive index ratio of the Malvern RTsizer was set according to a test medium, and a background measurement was performed before each test.

The value corresponding to $\mathrm{x}$ percentage finer size is termed as $D_{x} . D_{32}$ is the Sauter mean diameter used to monitor the fine particle, which is defined as:

$D_{32}=\frac{d_{1}^{3}+d_{2}^{3}+d_{3}^{3}+\cdots}{d_{1}^{2}+d_{2}^{2}+d_{3}^{2}+\cdots}$.
$D_{43}$ is the De Brouckere mean diameter used to monitor the coarser particle, which is defined as:

$D_{43}=\frac{d_{1}^{4}+d_{2}^{4}+d_{3}^{4}+\cdots}{d_{1}^{3}+d_{2}^{3}+d_{3}^{3}+\cdots}$.

ERT was used to measure the gas concentration of the gas-liquid two-phase flow (Singh et al., 2017; Sardeshpande et al., 2017; Jia et al., 2015). With the conductivity distribution from ERT, the gas concentration could be determined by applying the Maxwell relationship:

$\alpha_{g}=\frac{2 \sigma_{l}-2 \sigma_{m}}{2 \sigma_{l}+2 \sigma_{m}}$

where $\sigma_{l}$ is the conductivity of the liquid, and $\sigma_{m}$ is the local mixture conductivity.

Dual-plane ERT sensors were used in this study, and each ERT sensing plane consisted of 16 titanium-alloy rectangular electrodes. A total of 8000 dual-frames of voltage measurements were acquired for each flow condition, which took $8.75 \mathrm{~s}$ at a speed of 914.3 dual-frames per second. A sensitivity coefficient backprojection algorithm was adopted to reconstruct the flow images for its high speed, which adopted 316 square meshes. The reconstruction algorithm employed the voltage relative changes between a measurement profile acquired from a flow with gas concentration change and a reference profile acquired from a flow with pure water. Reference measurement error of $1 \%$ could lead to conductivity error of up to $10 \%$ depending on the magnitude of the conductivity charge (Wang et al., 1999). Before each test, the ERT system was calibrated, and the reference frame was took when the sensor was full of pure liquid so that the reference measurement error could be controlled within $1 \%$.

\subsection{Flow loop}

In the experiment, air was chosen as the gas phase. Tap water was used as the liquid phase. The flow loop is illustrated in Fig. 2. High-pressure water pumped from a tank and compressed air supplied by an air compressor were well mixed at the nozzle. A pressure-swirl nozzle was employed, which utilized centrifugal effect and the shear action between high-speed air flow and liquid to provide the finest atomized droplets. Subsequently, the atomized droplets were injected into the pipe, and compressed air that was provided by another air compressor transported the atomized droplets into the GLCC body rapidly. The inlet air was filtered for impurities, and then metered using a thermal gas mass flowmeter. The inlet water flow rate and flow rate of each phase in the mixture emerging from the liquid outlet were metered online using Coriolis mass flowmeters. The liquid flow rates obtained through the gas outlet and each AFE were separately collected and measured by the volumetric measurement technique. The pressures at the inlet and each outlet were monitored using pressure transmitters. All the output signals from the metering devices were acquired for over $40 \mathrm{~s}$, at reading intervals of $0.001 \mathrm{~s}$. The final measured quantity was calculated as the average of the 40-s data sets.

The atomized droplets were tangentially introduced into the GLCC body. Most of them ran into the lower part of the GLCC directly, while the rest moved upward with the high-speed air flow. A liquid film was formed in the upper part of the GLCC as the droplets pushed toward the wall by the centrifugal effect. Similar to the measurement of the droplet size distribution in the annular gas-liquid flow (Steimes and Hendrick, 2017), the droplet flow was extracted from the pipe before entering the measuring zone of the Malvern RTsizer, as shown in Fig. 3. The down sample 


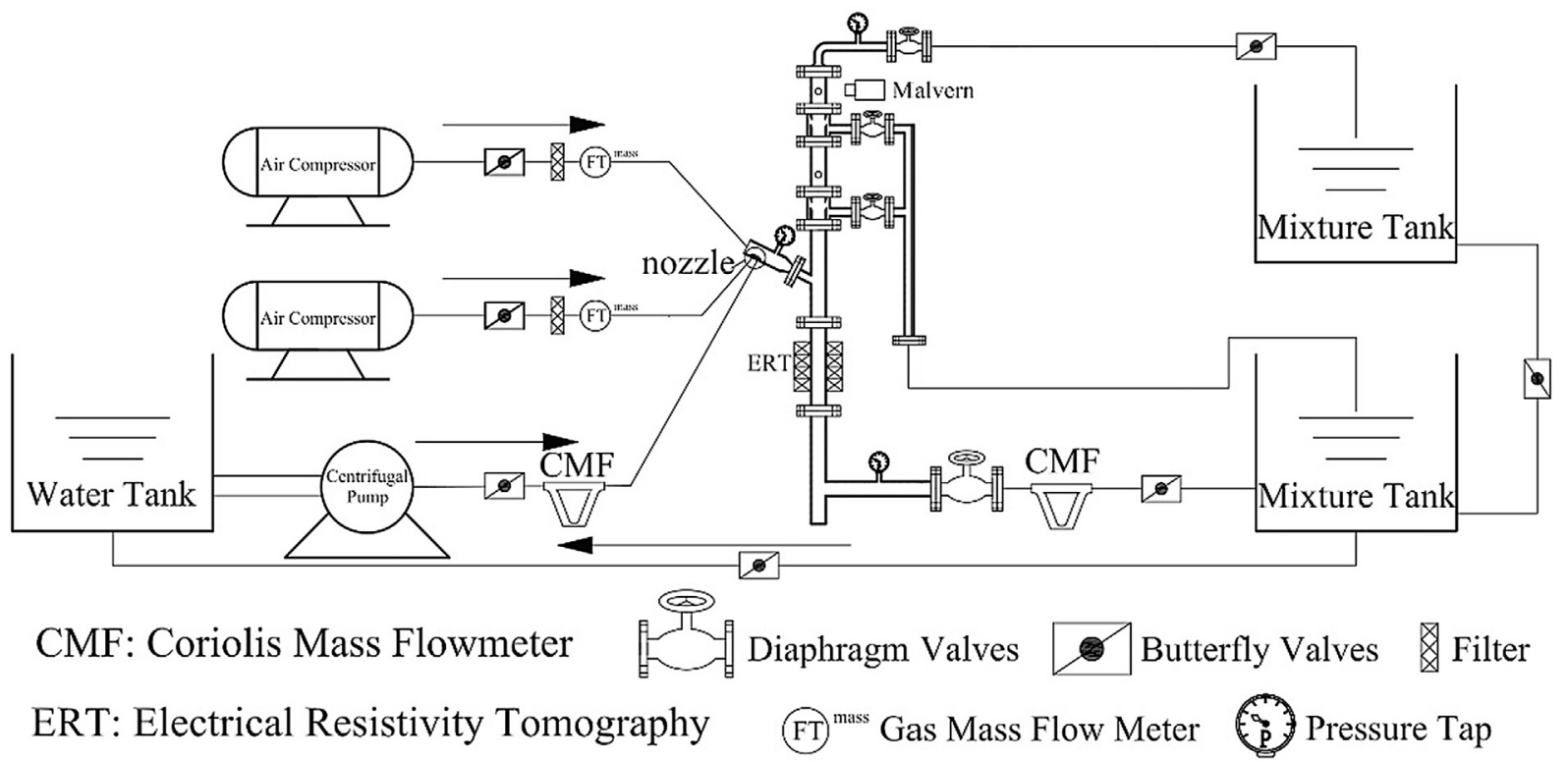

Fig. 2. Flow loop of the GLCC.

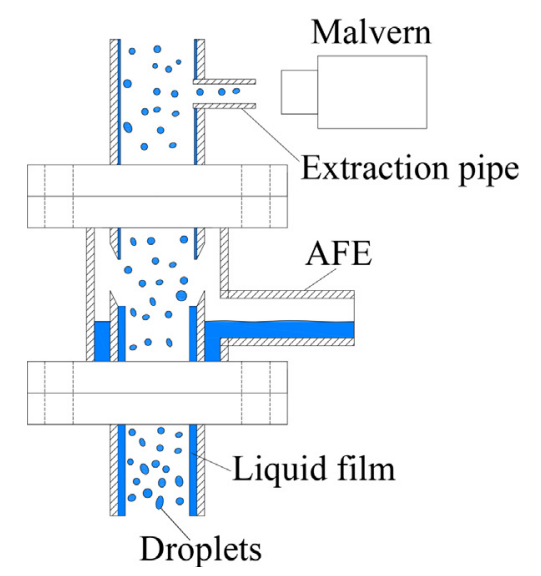

Fig. 3. Liquid film removal by AFE.

outlet (DSO) and the up sample outlet (USO) were located $410 \mathrm{~mm}$ and $725 \mathrm{~mm}$ above the inlet centerline, respectively. The liquid film was removed from the AFE, and an extraction pipe (I.D. $20 \mathrm{~mm}$ ) was fixed in the GLCC through a length of $2 \mathrm{~mm}$ to cross the potential new liquid film. In addition, the extraction pipe was short enough and its outlet was very close to the measuring zone to reduce the change of droplet distribution induced by other physical factors, such as gravitational deposition, breakage, and coalescence. In the lower part of the GLCC, dual-plane sensors of ERT 3000 were installed at positions $360 \mathrm{~mm}$ and $410 \mathrm{~mm}$ below the inlet centerline to measure the phase distribution in the swirling field.

\subsection{Experimental conditions}

All the experiments were carried out at a temperature of $20^{\circ} \mathrm{C}$. The experimental conditions were as follows. The density of tap water was $999.0 \mathrm{~kg} / \mathrm{m}^{3}$ with a viscosity of $1.0 \mathrm{mPa} \cdot \mathrm{s}$, while the density of air was $1.205 \mathrm{~kg} / \mathrm{m}^{3}$ with a viscosity of $1.81 \times 10^{-3} \mathrm{mPa} \cdot \mathrm{s}$. The air-water surface-tension coefficient was $0.072 \mathrm{~N} / \mathrm{m}$. For each inlet structure, 55 sets of inlet conditions were employed. The gas superficial velocity ranged from $12 \mathrm{~m} / \mathrm{s}$ to $64 \mathrm{~m} / \mathrm{s}$ and the liquid superficial velocity from $0.085 \mathrm{~m} / \mathrm{s}$ to $0.555 \mathrm{~m} / \mathrm{s}$, thereby covering a wide range of liquid volume fraction between $0.001 \%$ and $4.51 \%$.

\section{Numerical simulations}

\subsection{Numerical method}

In this study, numerical simulations were conducted by ANSYS Fluent 14.5. The DPM model that considers the impact of droplets breakup and coalescence on the separation process was developed to obtain the distribution characteristics of the entire flow field and the migration trajectories of the droplets in the GLCC. The continuous phase was described by the Euler method, and the time-averaged N-S equation was solved to obtain the flow field parameters. The discrete phase was described by the Lagrange method, and the trajectory was obtained by integrating the motion equation of several particles. The motion equation of a dispersed particle in the Lagrangian reference system is obtained according to the force analysis and can be written as:

$\frac{d u_{p}}{d t}=f_{D}\left(u-u_{p}\right)+\frac{\left(\rho_{p}-\rho\right) g_{x}}{\rho_{p}}+f_{x}$

where $f_{D}\left(u-u_{p}\right)$ is the drag force per unit particle mass; $\left(\rho_{p}-\rho\right) g_{x} / \rho_{p}$, the resultant force of gravity and buoyancy; $f_{x}$, an additional acceleration term; $f_{D}$, the drag coefficient; $u$, the continuous phase velocity; $u_{p}$, the discrete phase velocity; $\rho$, the continuous phase density; and $\rho_{p}$, the discrete phase density.

O'Rourke (1981) estimated the collision frequency using the stochastic method. He assumed that collisions may occur only when two parcels existed in the same grid. When the grid is smaller than the droplet size, this method has a second-order accuracy. Based on the concept of collision volume, the collision frequency can be deduced as:

$P_{1}=\frac{\pi\left(r_{1}+r_{2}\right)^{2} v_{r e l} \Delta t}{V_{c}}$

where $r_{1}$ and $r_{2}$ are the radii of the two parcels. $V_{c}$ is the volume of the grid, and $v_{\text {rel }}$ is the relative velocity between the two parcels.

After evaluating the collision of the two parcels, it is necessary to further determine the results of their collision. O'Rourke 
assumed that two parcels coalesced when they collided frontally and rebounded off when they collided sideways. The critical collision parameters are as follows:

$b_{\text {crit }}=\left(r_{1}+r_{2}\right) \sqrt{\min \left(1.0, \frac{2.4 f}{W e}\right)}$

where $f$ is a function of $r_{1} / r_{2}$, and We is the collision Weber number.

The actual collision parameter, $b$, is shown as Eq. (8):

$b=\left(r_{1}+r_{2}\right) \sqrt{Y}$

where $Y$ is the mean variation.

It is assumed that part of the lost kinetic energy is converted into viscous dissipation and angular momentum. The rate of energy loss is correlated with the collision parameter. Based on this energy loss assumption, O'Rourke derived the parcel velocity after the collision as follows:

$v_{1}^{\prime}=\frac{m_{1} v_{1}+m_{2} v_{2}}{m_{1} m_{2}}+\frac{m_{2}\left(v_{1}-v_{2}\right)}{m_{1} m_{2}}\left(\frac{b-b_{\text {crit }}}{r_{1}+r_{2}-b_{\text {crit }}}\right)$

To reasonably describe the dynamic characteristics of the droplets, a droplet breakup model was applied to simulate the entire breakup process of the droplet. The classical droplet breakup model is a wave-breaking model proposed by Reitz (1987), which is suitable for simulating the droplet breakup process when the Weber number is greater than 100 . The model considers that the velocity difference with respect to the gas phase and liquid phase causes the droplet to breakup. The breakup time and the droplet size after the breakup are associated with fast-growing KelvinHelmholtz unstable surface wave. Another common droplet breakup model is the TAB model, which is suitable for simulating the droplet breakup process when the Weber number is less than 100. Given that the Weber number in this study is less than 100 , the TAB model is a suitable option. The TAB model describes the droplet breakup process through the critical deformation of oscillating droplets. The relative motion of the droplet provides the external force required for the vibration. The surface tension of the droplet acts as the restoring force to prevent the liquid from vibrating. The viscous force of the droplet acts as a damping force to prevent the vibration deformation of the droplet.

The forced damped vibration of the droplet can be expressed using the following governing equation:

$\frac{d^{2} y}{d t^{2}}=\frac{2 \rho_{g} U_{m}^{2}}{3 \rho_{l} r_{d}^{2}}-\frac{8 \sigma}{\rho_{l} r_{d}^{3}} y-\frac{5 \mu_{l}}{\rho_{l} r_{d}^{2}} \frac{d y}{d t}$

where $y=2 e / r_{d}$ is the normalized radial deformation of the droplet. In the formula, $e$ is the deformation amount of droplet in the radial direction, and $r_{d}$ is the radius of the droplet.

According to the energy conservation before and after the droplet breakup, the droplets sizes after the breakup can be calculated. The droplet distribution can be expressed by the Sauter mean diameter:

$r_{32}=\frac{r_{p}}{\frac{7}{3}+\frac{\rho_{l} l_{p}^{3}(d y / d t)_{b u}^{2}}{8 \sigma}}$

where $r_{p}$ is the droplet diameter before the breakup, and $(d y / d t)_{b u}$ is the value of $d y / d t$ at $y=1$. The droplet size distribution after the breakup can be obtained using the TAB model as:

$f\left(r_{d}\right)=\frac{3}{r_{32}} \exp \left(-\frac{3 r_{d}}{r_{32}}\right)$

The RNG $k-\varepsilon$ model considers both turbulent vortices and the time-averaged strain rate of the mainstream. It can achieve better results in simulating a flow with large streamline curvature and high strain rate. The transport equations are as follows: $\frac{\partial}{\partial t}(\rho k)+\frac{\partial}{\partial x_{i}}\left(\rho k u_{i}\right)=\frac{\partial}{\partial x_{j}}\left(\alpha_{k} \mu_{e f f} \frac{\partial k}{\partial x_{j}}\right)+G_{k}+G_{b}-\rho \varepsilon-Y_{M}+S_{k}$

$$
\begin{aligned}
\frac{\partial}{\partial t}(\rho \varepsilon)+\frac{\partial}{\partial x_{i}}\left(\rho \varepsilon u_{i}\right)= & \frac{\partial}{\partial x_{j}}\left(\alpha_{k} \mu_{e f f} \frac{\partial \varepsilon}{\partial x_{j}}\right)+C_{1 \varepsilon} \frac{\varepsilon}{k}\left(G_{k}+C_{3 \varepsilon} G_{b}\right) \\
& -C_{2 \varepsilon} \rho \frac{\varepsilon^{2}}{k}-R_{\varepsilon}+S_{\varepsilon}
\end{aligned}
$$

The current literature shows that no turbulence model performs better for all kinds of swirl flows (Hreiz et al., 2011). Comparison with the experimental results is the best way to choose proper turbulent model. The RNG $k-\varepsilon$ model has been utilized to describe the turbulent flow process in the GLCC (Yue et al., 2019; Erdal et al., 1996; Han et al., 2013; Gupta and Kumar, 2007). In these literatures, the simulated pressure, velocity, and film height agreed well with measured values, which verified the effectiveness of the RNG $k-\varepsilon$ model for the numerical simulation of the GLCC. Their simulation conditions are similar to those in this study. In this study, the RNG $k-\varepsilon$ model was also employed to simulate the turbulent flow in the GLCC. In Section 3.4, the comparison between numerical and experimental results indicates that RNG $k-\varepsilon$ model is acceptable for simulating the gas-liquid two-phase flow in the GLCC.

\subsection{Boundary conditions}

The gas inlet adopts a uniform velocity inlet boundary. The pressure outlet was set according to the measured pressure value in the experiment. For the dispersed liquid phase, the liquid mass flow rate was set at the inlet. The Rosin-Rammler distribution was used to characterize the inlet droplet size distribution measured using the Malvern RTsizer as the inlet boundary conditions of the dispersed droplets, as shown in Fig. 4. The wall function method is adopted to evaluate the boundary condition at the standing wall.

\subsection{Geometrical model and meshing}

Fig. 5 is the geometrical model and the mesh of the GLCC. The structure dimension of the model is consistent with that of an experimental device. To improve the computational accuracy, the grid was divided into blocks. The flow field and phase distribution at the inlet were the most complex. Thus, the compact unstructured grid was used at the inlet. The structured grid was adopted in the rest. According to the verification of the grid independence, a grid with $1,557,350$ cells was chosen as the final grid scheme.

\subsection{Verification of simulation}

The droplets gathered around the wall, permitting the visualization of the flow field. Fig. 6 shows the streamline distribution obtained by experimental observation and numerical simulation when the gas superficial velocity was $49 \mathrm{~m} / \mathrm{s}$ and the liquid superficial velocity was $0.283 \mathrm{~m} / \mathrm{s}$. As can be seen, the fluid was divided into two paths at the inlet. Numerical results clearly indicated that helical motion was adopted in the upper part of the GLCC. According to the experimental photograph, the liquid was driven into the upper part of the GLCC by the high-speed air flow and the forming liquid film moved upward spirally. Through numerical simulation and experimental observation, it was found that the flow field in the lower part of the GLCC exhibited an abnormal disorder due to the gas reverse movement. In summary, through qualitative comparison, the streamline distribution predicted by numerical simulation was, basically, consistent with that observed by experiment. 

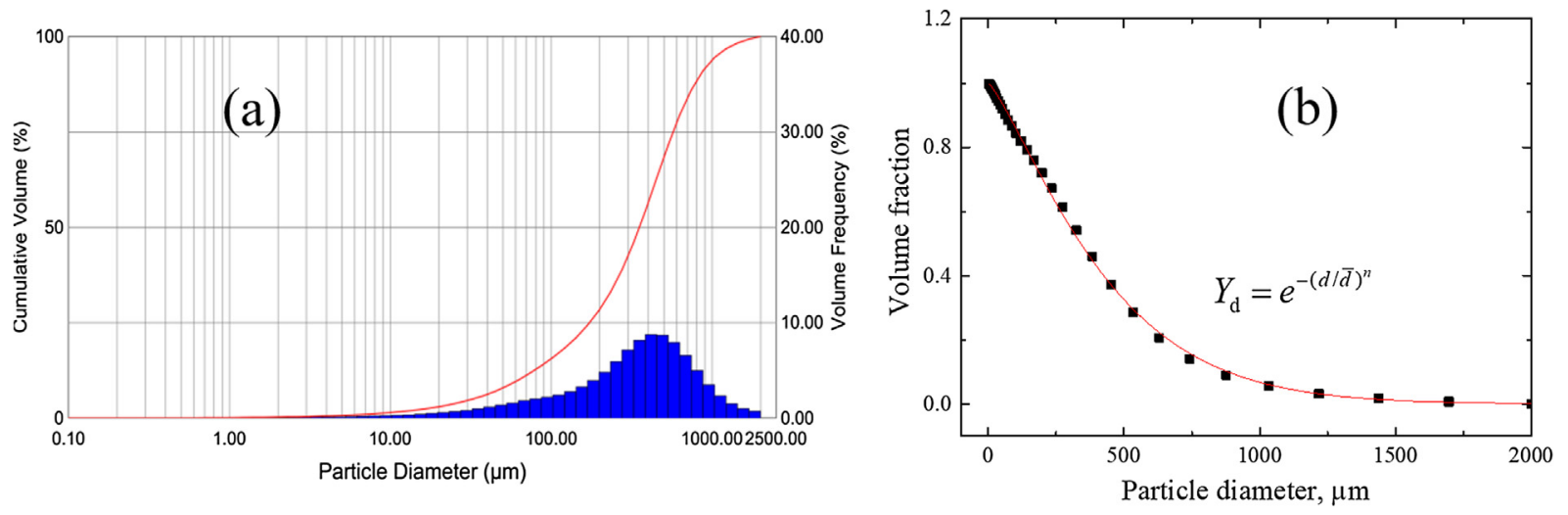

Fig. 4. Inlet droplet size distribution (a) measured using the Malvern RTsizer, (b) described by Rosin-Rammler.

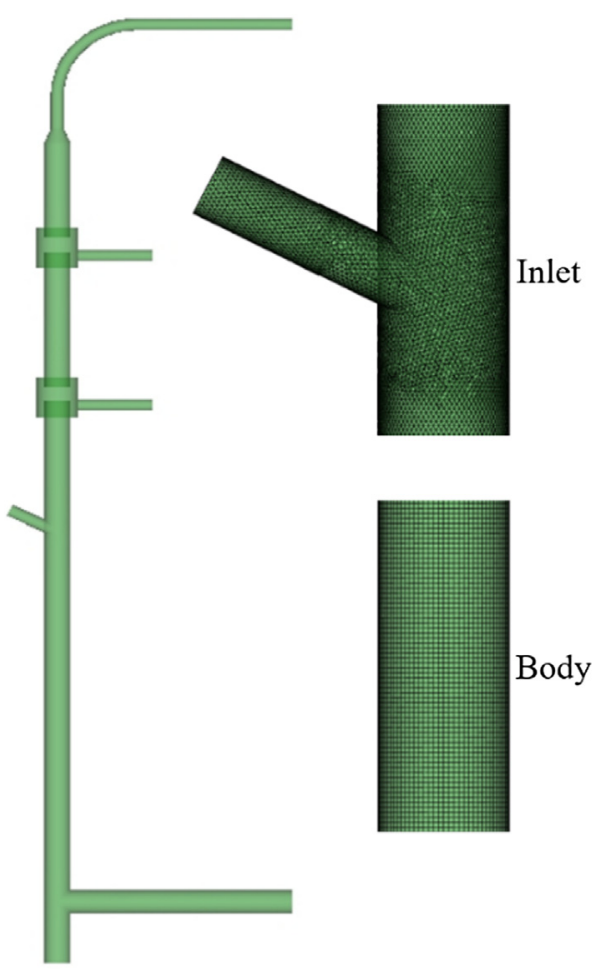

Fig. 5. Geometrical model and mesh of the GLCC.

The mixed Reynolds number is defined as:

$\operatorname{Re}_{m}=\frac{\rho_{m} v_{m} D}{\mu_{m}}$

where $v_{m}, \rho_{m}$, and $\mu_{m}$ are the gas-liquid mixture velocity, density, and viscosity, respectively. All these parameters are assumed as the weighted average of the phase volume fraction.

The variation of the pressure drop with the mixed Reynolds number obtained by numerical simulation is in agreement with the experiment results, as shown in Fig. 7a. With the increase in the mixed Reynolds number, the pressure drop increases approximately linearly. Fig. 7b shows that the simulated droplet size distribution at the DSO agrees well with the Malvern sampling result. Therefore, the numerical model developed in this study is adequate to predict the gas-liquid separation behavior and hydrodynamic characteristics in the GLCC.
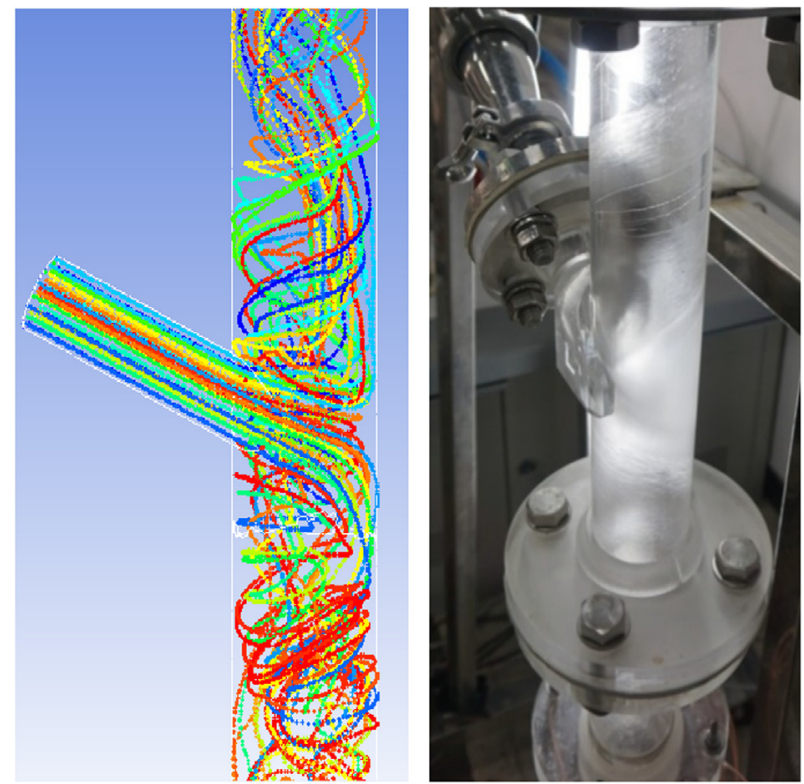

Fig. 6. Comparison between the streamlines by experimental observation and by numerical simulation.

\section{Results and discussion}

\subsection{Liquid carry-over}

\subsubsection{Effect of the liquid level on liquid carry-over}

The liquid level is an important parameter for gas-liquid separation performance. In this work, the liquid level was controlled by adjusting the differential pressure between the upper and lower outlets. To evaluate the separation efficiency, the upper liquid carry-over was defined as the liquid extracted from each AFE and emerging from the gas outlet. Variations of characteristic sizes and the upper liquid carry-over with respect to the liquid level were investigated, as shown in Figs. 8 and 9. When the liquid level was below the inlet and above the liquid outlet, the droplet size distribution and the amount of the upper liquid carry-over were independent of the liquid level. This is because the lower part of the GLCC was almost a "dead zone" for the gas phase in this situation. The liquid level provided a barrier against the downward flow of the gas. Thus, almost the entire volume of gas flowed directly into the upper part of the GLCC from the inlet. The variation of the liquid level had a negligible influence on the flow field distribution in the upper part of the GLCC. When the liquid level 

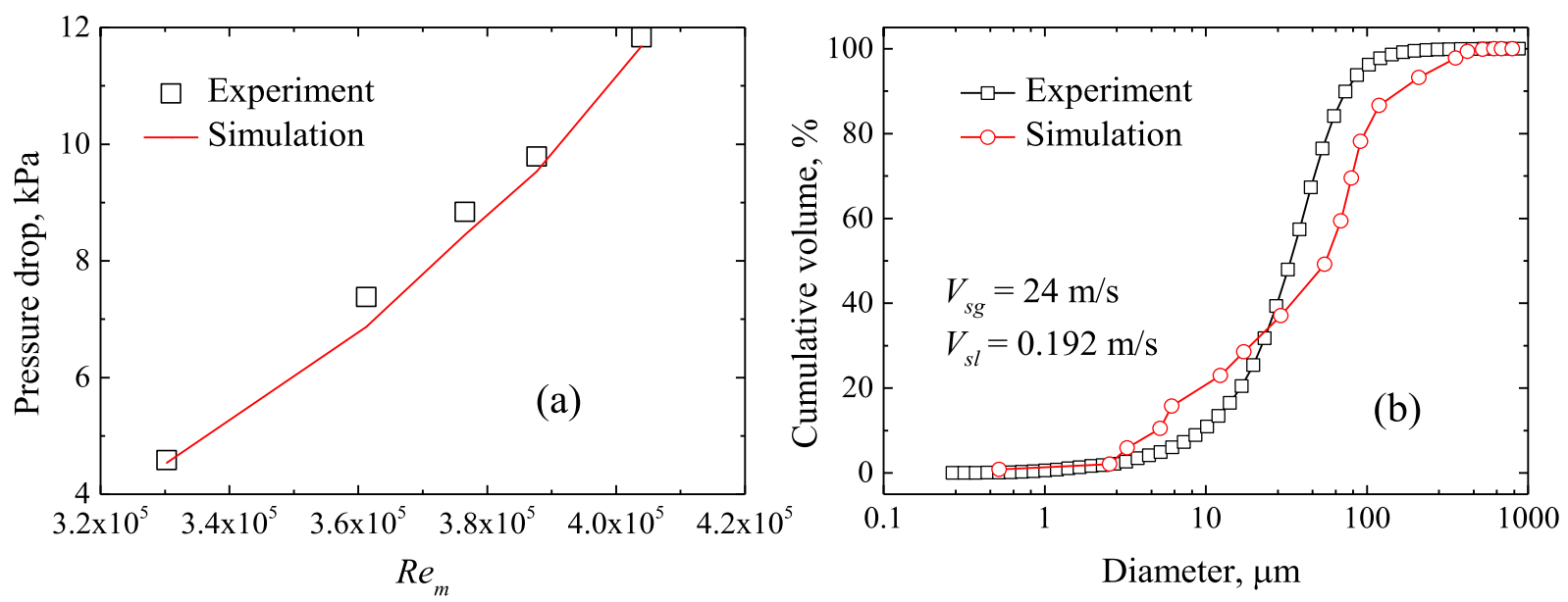

Fig. 7. Comparison between the experimental data and numerical simulation for the pressure drop (a) and droplet size distribution at the DSO (b).

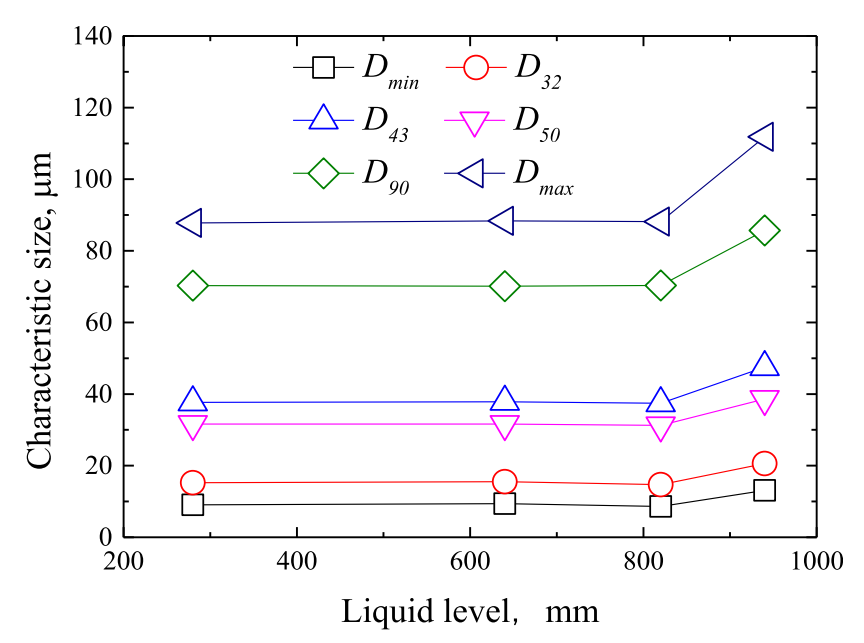

Fig. 8. Effect of the liquid level on the droplet size distribution at the down sample outlet.

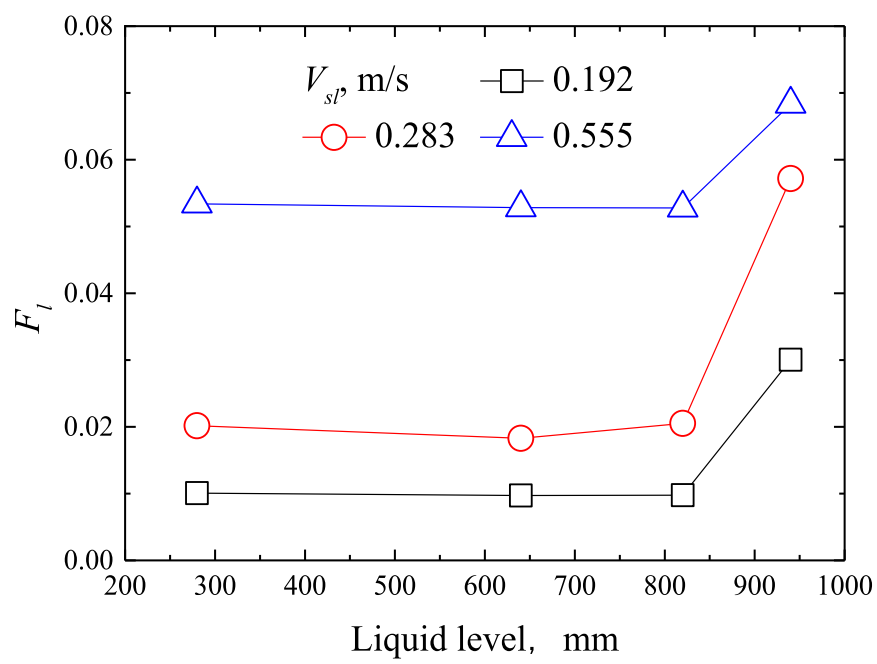

Fig. 9. Effect of the liquid level on the upper liquid carry-over.

was above the inlet, large droplets were formed under the shear action of high-speed gas flow on the liquid phase. These droplets moved upwards with the high-speed gas flow. Thereafter, the characteristic sizes and the amount of the upper liquid carryover increased dramatically. This increase should be avoided in gas-liquid separation. Therefore, this work mainly studied the flow characteristics in the GLCC when the liquid level was below the inlet and above the liquid outlet.

\subsubsection{Effect of the gas-liquid flow rates on the liquid carry-over}

The upper liquid carry-over ratio is defined as the ratio of the amount of the upper liquid carry-over to the inlet liquid flow rate:

$F_{l}=\frac{Q_{u l}}{Q_{l}}$

where $Q_{u l}$ is the amount of the upper liquid carry-over and $Q_{l}$ is the inlet liquid flow rate.

Fig. 10a shows the change in the upper liquid carry-over ratio with the gas superficial velocity. As can be seen, the upper liquid carry-over ratio is zero for a low gas superficial velocity. This is because almost all the droplets directly entered the lower part of the GLCC, and eventually flowed away from the liquid outlet. A considerably small amount of droplet entered the upper part of the GLCC. Due to the weak carrying capacity of the low flowrates gas stream, these droplets quickly fell back to the lower part of the GLCC under gravity. There was no liquid flowing away from the gas outlet. With the increase in the gas superficial velocity, the upper liquid carry-over ratio increased firstly and then decreased. As the gas superficial velocity increased, the carrying capacity of the gas stream improved gradually. Gradually, the gas stream was able to overcome the gravity effect of the droplets and carried them upward. The larger the gas superficial velocity, the more droplets can be transported. Due to a relatively weak swirl intensity, the droplets had to travel a long distance in the axial direction of the GLCC before they reached the wall to form a liquid film. Therefore, the liquid film formed by centrifugation in the upper part of the GLCC was usually thin in this situation. As the gas superficial velocity increased further, the swirl intensity began to increase rapidly. The droplets could quickly migrate to the wall to form a thick liquid film above the inlet, which was difficult for high flow-rate gas streams to carry upward. Therefore, the thick liquid film rapidly fell back to the lower part of the GLCC before reaching the AFE. This can lead to a gradual decrease in the upper liquid carry-over ratio.

The effect of the liquid superficial velocity on the upper liquid carry-over ratio is depicted in Fig. 10b. The upper liquid carryover ratio increases as the liquid superficial velocity decreases. This is because the atomization effect of the inlet nozzle becomes more pronounced as the liquid superficial velocity decreases. The 

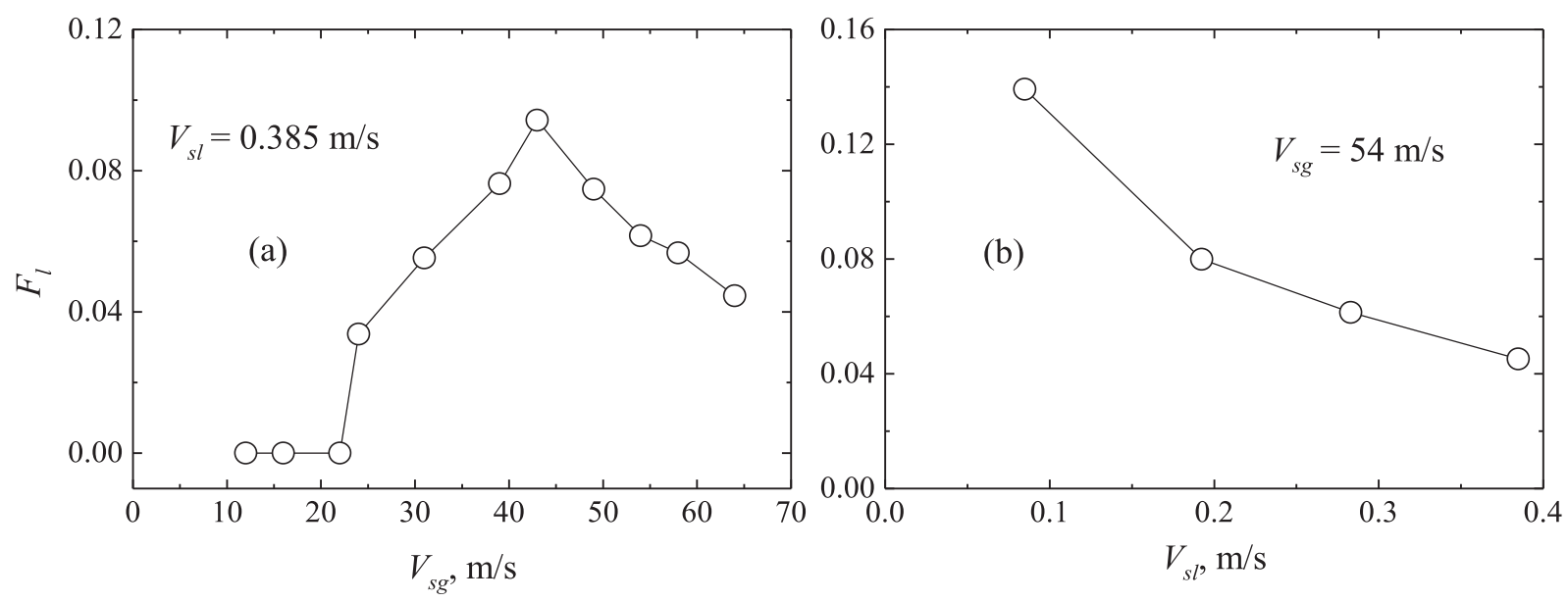

Fig. 10. Effect of the gas (a) and liquid (b) superficial velocity on the upper liquid carry-over ratio.

proportion of small droplets to the total inlet liquid volume increases. It became easier for small droplets to be transported upward by high flow-rate gas streams, which resulted in the liquid carry-over.

\subsubsection{Effect of the necking ratio on liquid carry-over}

The effect of the necking ratio on the upper liquid carry-over ratio is demonstrated in Fig. 11. As can be seen, the initial gas superficial velocity leading to the liquid carry-over for the nozzle with $\mathrm{Ne}=4.0$ is much lower than that for the other two inlet structures. The main reason is that the swirl intensity is low when the necking ratio is 4.0. Consequently, the droplets do not rapidly migrate to the wall to form a liquid film. Therefore, the liquid phase mainly exists in the form of droplets above the inlet. Low flow-rate gas streams can transport these droplets upward, as shown in Fig. 12a. With the increase in the necking ratio, the droplets can rapidly migrate to the wall to form a liquid film. For the nozzle with $\mathrm{Ne}=5.9$, the swirl intensity is moderate, and the shear action of the swirling gas flow on the liquid film is relatively weak. As shown in Fig. 12b, the liquid film can maintain a relatively stable shape, which can be carried upward only by high flowrates gas streams. Therefore, the initial gas superficial velocity leading to the liquid carry-over for the nozzle with $\mathrm{Ne}=5.9$ was high. However, the necking was too severe for the nozzle with $\mathrm{Ne}=10.7$. When the gas superficial velocity increased to a certain extent, strong swirling flow caused strong shear action on the upward moving liquid film. As shown in Fig. 12c, a large number

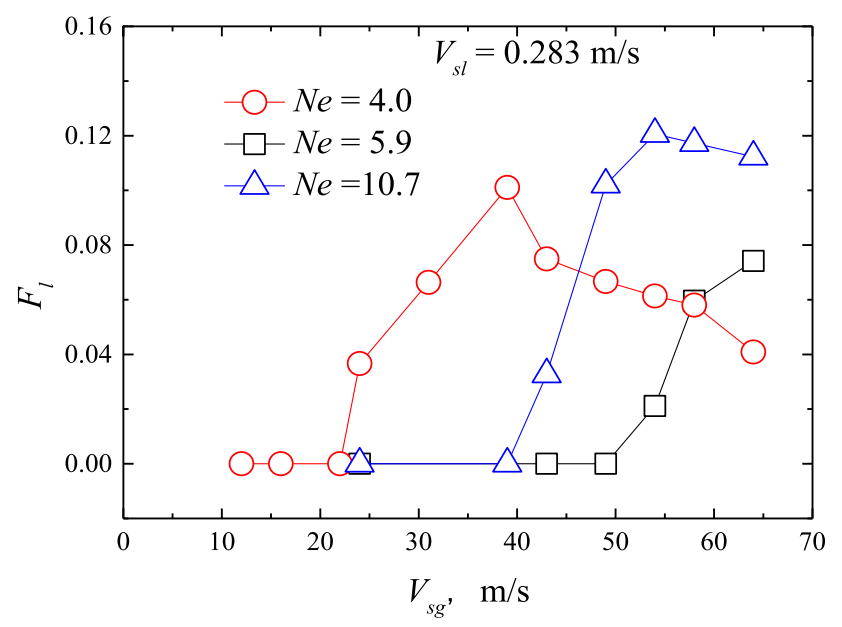

Fig. 11. Upper liquid carry-over ratio for different necking ratios. of new droplets were formed, resulting in a significant increase in the upper liquid carry-over ratio. In general, the nozzle with $\mathrm{Ne}=5.9$ performs best for separating the liquid from the gas flow. Combining with the results about the effect of the gas superficial velocity on the upper liquid carry-over ratio, it can be concluded that the separator with strong swirl intensity will not necessarily get better separation performance.

\subsubsection{Breakup, coalescence, and migration regularity of the droplets}

The droplet size distributions at the inlet and both sample outlets are shown in Fig. 13. The droplet size distributions at both sample outlets are obviously smaller than that at the inlet, while the droplet size distribution and characteristic size at the up sample outlet are slightly smaller than those at the down sample outlet are. This shows that the gas-liquid separation mainly occurs at the inlet and between the inlet and the down sample outlet. The gasliquid separation effect is not obvious between two sample outlets, which is basically consistent with the observed experimental phenomena (Fig. 14).

Fig. 15 depicts the droplets distributions at different times with a gas superficial velocity of $49 \mathrm{~m} / \mathrm{s}$ and liquid superficial velocity of $0.283 \mathrm{~m} / \mathrm{s}$. In the figure, blue represents the sizes of small droplets and red represents the sizes of large droplets. The droplets in the lower part of the GLCC are considerably large; consequently, the color of the droplets larger than $3 \mathrm{~mm}$ is set to be the same with that of $3 \mathrm{~mm}$ droplets to make the differences between small droplets and medium droplets more intuitive. At the inlet, most droplets, particularly those with relatively large particle sizes, directly enter the lower part of the GLCC due to gravity and inertia. As can be clearly seen from Fig. 15, these droplets exhibit threedimensional helical motions. Due to centrifugal action, the probability of collision and coalescence of the droplets near the wall is larger than that in other areas, and the formed droplets near the wall are the largest. Only a small number of droplets, particularly those with relatively small particle sizes, move upward with the high-speed airflow. These droplets can maintain good coherence with the gas and have the highest migration speed. Thus, they enter and fill the upper part of the GLCC quickly. However, these droplets can migrate to the wall to form a liquid film under the centrifugal force, and relatively large droplets are easy to migrate to the wall to form a liquid film. The liquid film flows out from the down annular film extractor. Only small droplets are left in the GLCC. The centrifugal force of these small droplets is low. Simultaneously, due to the rapid attenuation of the swirl intensity above the inlet, the swirl intensity between the down sample outlet and the up sample outlet was significantly weakened compared 


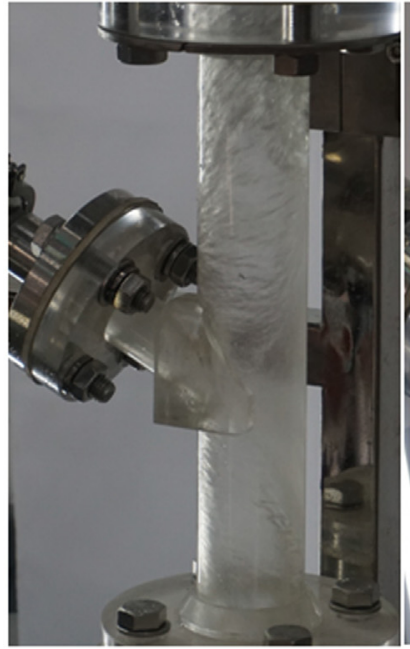

Droplets

(a) $\mathrm{Ne}=4.0$

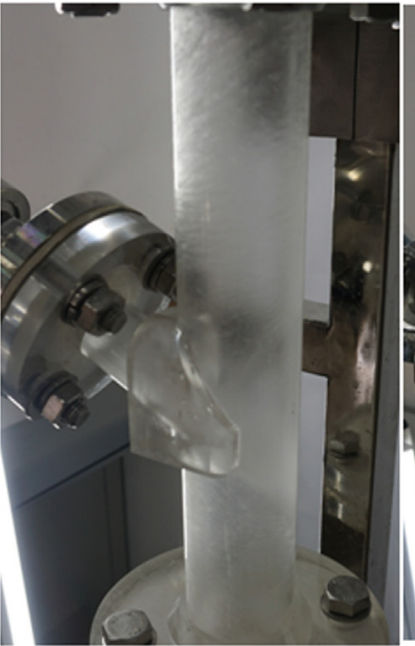

Steady liquid film

(b) $\mathrm{Ne}=5.9$

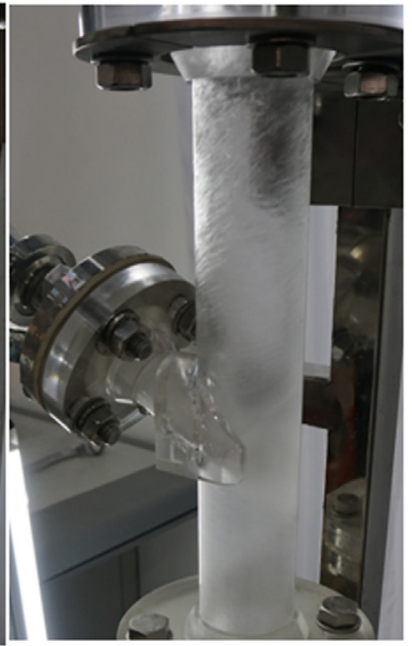

Broken liquid film

(c) $N e=10.7$

Fig. 12. Liquid films for the different necking ratios.

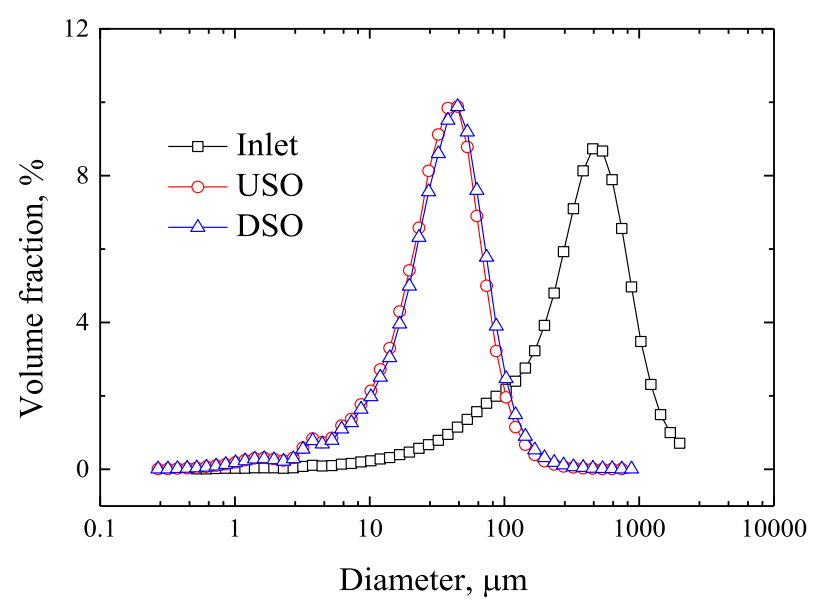

Fig. 13. Measured droplet size distributions at the inlet and both sample outlets.

with that at the inlet. It is difficult to throw these small droplets to the wall.

Fig. 16 illustrates the effect of the gas superficial velocity on the characteristic size at the down sample outlet with a liquid superficial velocity of $0.283 \mathrm{~m} / \mathrm{s}$. With the increase in the gas superficial velocity, $d_{\min }, d_{32}$, and $d_{50}$ show the same trend, namely, first increasing and then decreasing. According to Zhang and $\mathrm{Xu}$ (2016), the increase of $d_{32}$ implies a decrease in the turbulent dissipation energy. Relatively low turbulent dissipation implies a more ordered flow, and the augmentation of the gas superficial velocity contributes to the improvement of the swirl intensity. The probability of collision and coalescence between small and medium droplets increases correspondingly. Thus, $d_{\min }$ and $d_{50}$ first increase with the increase in the gas superficial velocity. However, as the gas superficial velocity increases further, the shear damage of small and medium droplets owing to the continuous gas phase is greater than the coalescence caused by the enhancement of the swirl intensity. The decline of $d_{32}$ indicates that the disorder degree of the swirling flow field increases. The coalescence effect of the droplets is weakened in this disordered flow field. Thus, the overall breakup effect outweighs the coalescence effect. In summary, small and medium droplets initially tend to coalesce,

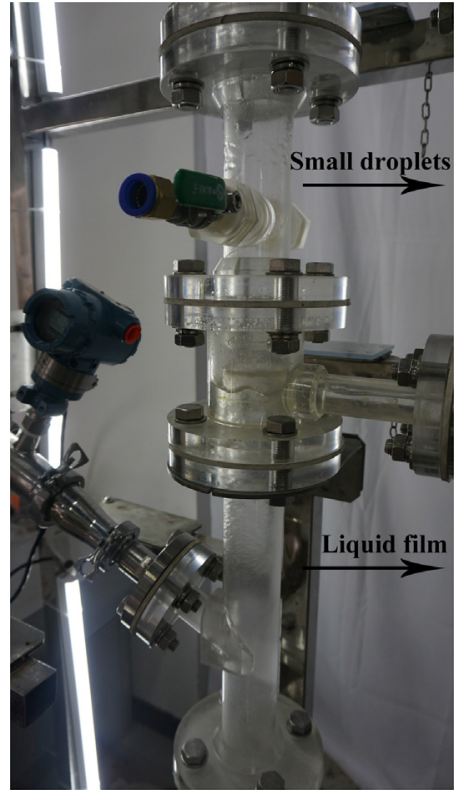

Fig. 14. Variation of the droplet size distribution in the upper part of the GLCC.

and subsequently breakup with the increase in the gas superficial velocity.

Different from other characteristic sizes, the maximum droplet diameter at the down sample outlet decreases gradually with the increase in the gas superficial velocity, and this works for two reasons. On the one hand, the increase in the gas superficial velocity improves the swirl intensity and the droplets, which can be separated, are smaller. On the other hand, there exists a maximum stable droplet diameter in the swirling flow field. Hinze (1955) investigated the breakup of dispersed droplets in a turbulent isotropic flow field. He believed that the action time of the turbulent stress that results in the breakup of droplets was short. The breakup of the droplets was mainly caused by the inertia force. The Weber number was used to characterize the influence of the shear force and surface tension of the continuous phase on the droplet breakup, and was regarded as the critical condition for the 


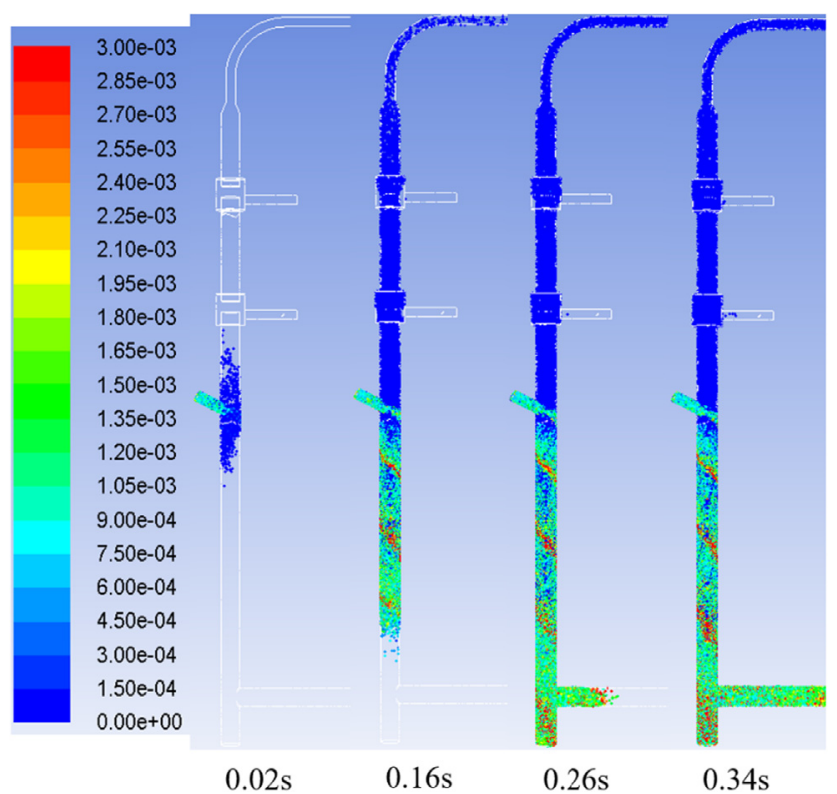

Fig. 15. Simulated droplet size distribution at different times (unit: $\mathrm{m}$ ).

droplet breakup. The maximum stable droplet size can be calculated using the critical Weber number.

$\frac{\rho_{\mathrm{c}} v^{2} D_{\max }}{\sigma}=\mathrm{const}$
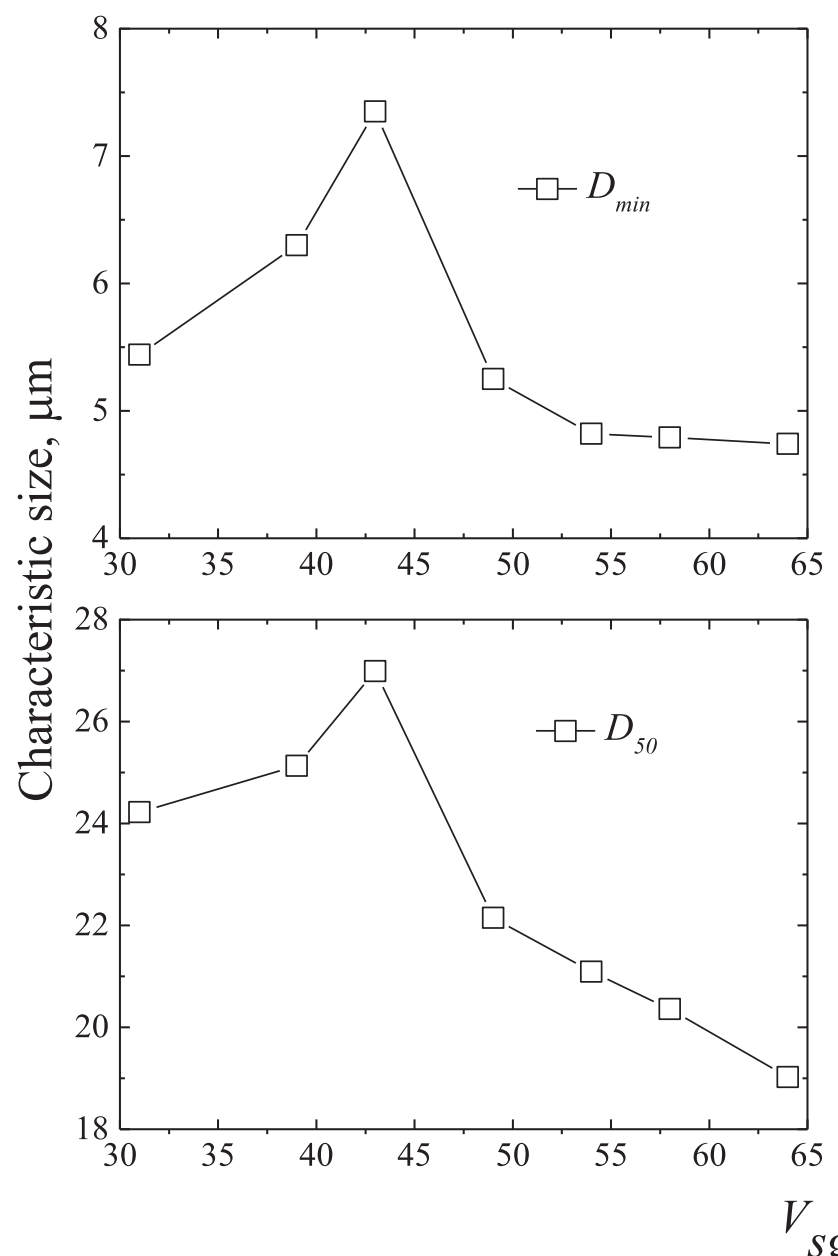

where $\rho_{c}$ is the density of the continuous phase; $v$ is the maximum slip velocity; and $\sigma$ is the surface tension coefficient.

The two-phase slip velocity increases with the continuous phase velocity. Subsequently, the shear damage of small and medium droplets owing to the continuous gas phase becomes more significant. According to Eq. (17), the maximum stable droplet size decreases. These two factors lead to a decreased maximum droplet size at the down sample outlet as the gas superficial velocity increases.

Fig. 17 illustrates the effect of the liquid superficial velocity on the characteristic size at the down sample outlet with a gas superficial velocity of $43 \mathrm{~m} / \mathrm{s} . d_{\min }, d_{32}$, and $d_{50}$ increase with the liquid superficial velocity. This indicates that small and medium droplets tend to coalescence. According to the study on the characteristics of the droplet size distribution at the inlet in Section 4.1, it was found that the increase in the liquid superficial velocity led to an increase in the sizes of the small droplets. The number of droplets entering the GLCC also increased. Meanwhile, increasing $d_{32}$ implies the decrease in the disordered degree of the swirling field. All these can increase the probability of collision between small droplets and medium droplets, which, in turn, causes the coalescence effect to increase.

Unlike other characteristic sizes, the maximum droplet diameter at the down sample outlet varies slightly with the liquid superficial velocity. This is because the maximum droplet size at the down sample outlet is mainly determined by the upper swirling flow field, which is mainly determined by inlet gas superficial velocity and necking ratio. As discrete phases, the droplets weakly influence the upper swirling flow field alone. Therefore, the change
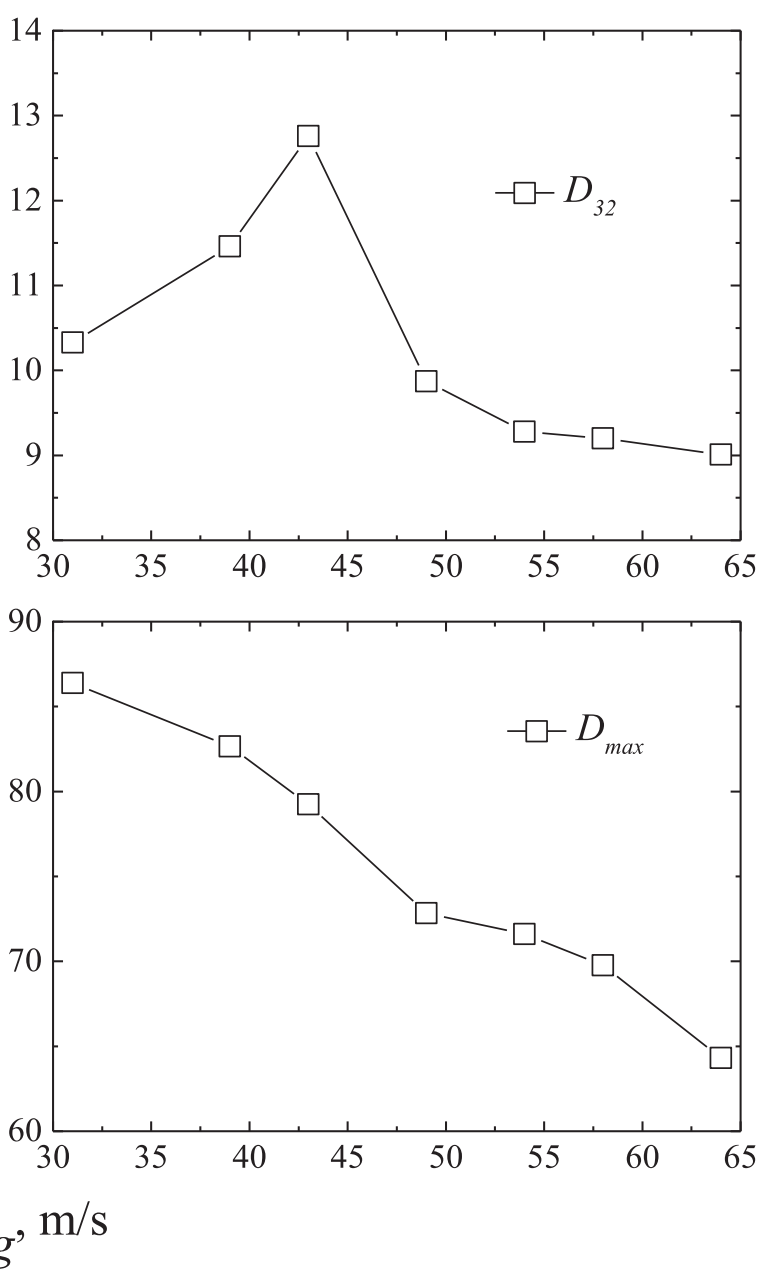

Fig. 16. Measured characteristic size at the down sample outlet for different gas superficial velocities under $V_{s l}=0.283 \mathrm{~m} / \mathrm{s}$. 


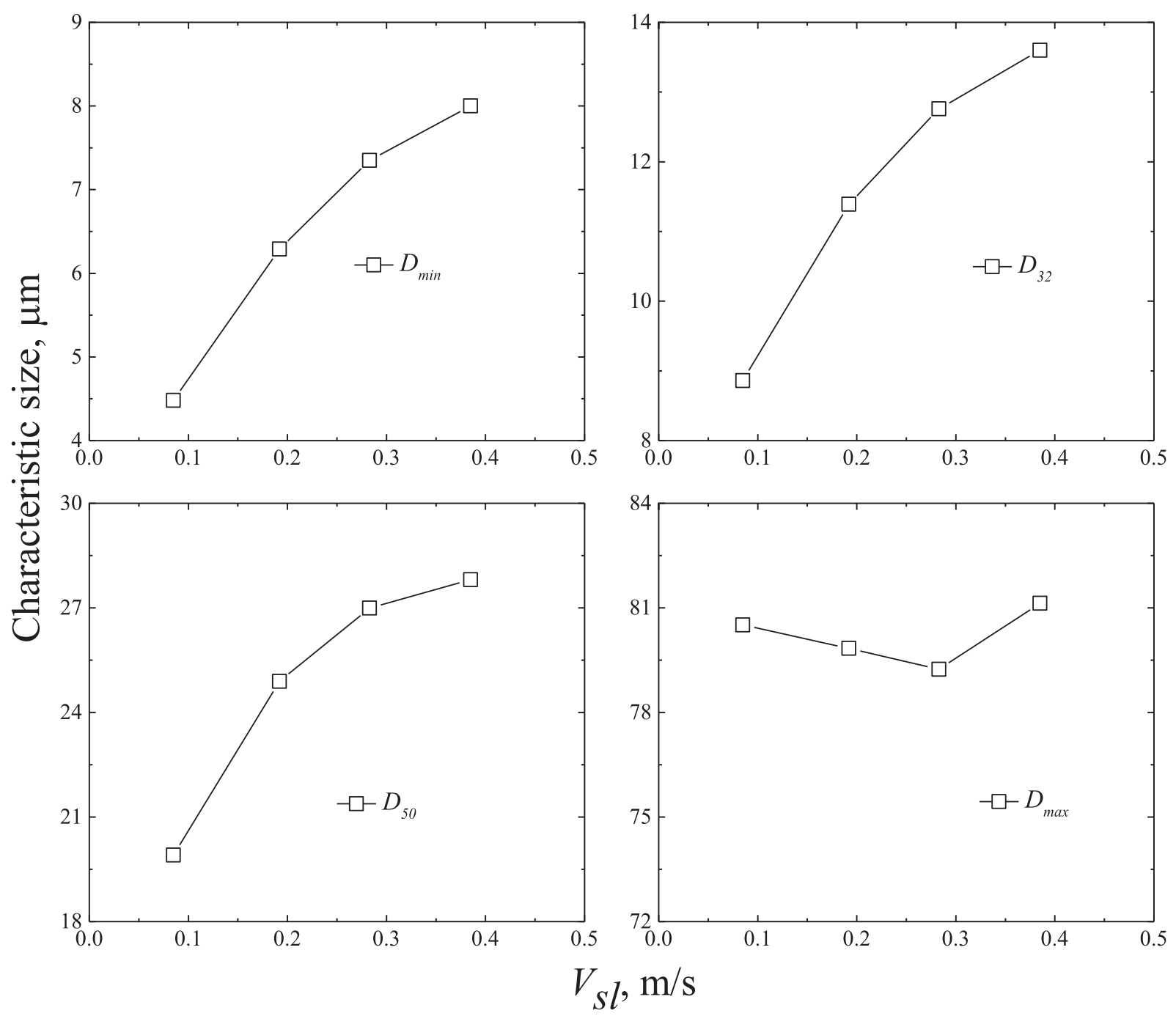

Fig. 17. Measured characteristic size at the down sample outlet for different liquid superficial velocities under $V_{\mathrm{sg}}=43 \mathrm{~m} / \mathrm{s}$.

in the liquid superficial velocity has little effect on the maximum diameter at the down sample outlet.

Fig. 18 shows the effect of the necking ratio on the maximum droplet diameter at the down sample outlet. It can be seen that the larger the necking ratio, the smaller the maximum droplet size at the down sample outlet. The necking ratio is an important parameter affecting the swirl intensity of the GLCC. A large necking ratio implies a high tangential velocity of the fluid entering the GLCC. The swirl intensity increases and the droplets that can be separated become smaller.

\subsection{Gas carry-under}

\subsubsection{Phase distribution in the lower part of the GLCC}

Fig. 19 shows the local void fraction in the lower part of the GLCC when the gas superficial velocity is $24 \mathrm{~m} / \mathrm{s}$ and the liquid superficial velocity is $0.385 \mathrm{~m} / \mathrm{s}$. As can be seen, the corresponding measuring results of ERT is basically consistent with the experimental observation. The average gas holdup in the upper section is $2.75 \%$, and the average gas holdup in the lower section is $1.93 \%$. The gas-liquid separation laws in the lower part of the GLCC can be obtained from these two figures. A large number of bubbles are formed at the liquid surface. With the rotation of the liquid phase, these bubbles are pushed radially toward the vortex center

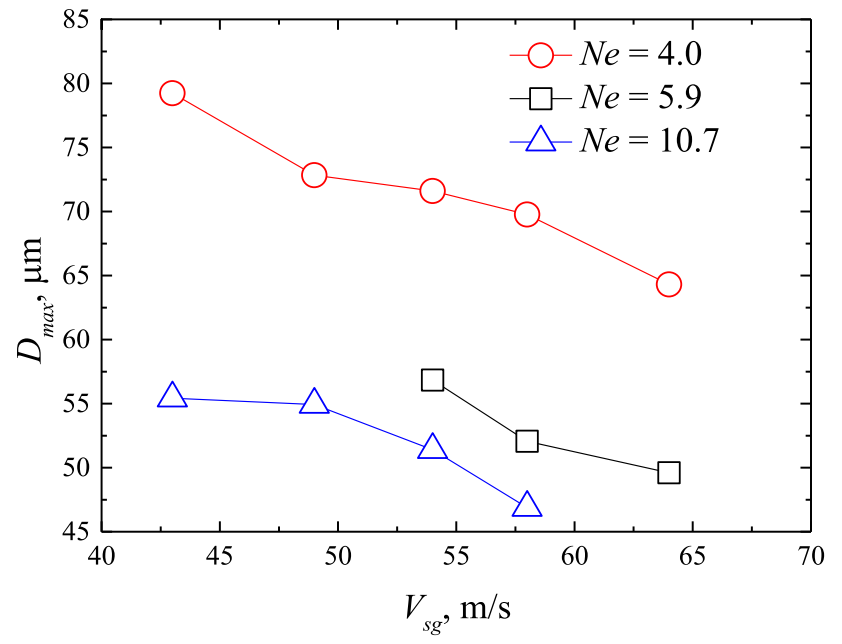

Fig. 18. Effect of the necking ratio on the maximum diameter at the down sample outlet.

and accumulated in the form of a gas core. Fig. 20 plots the local void fraction distributions along the pipe horizontal centerline, which is also measured by ERT. Due to a low inlet liquid velocity, 

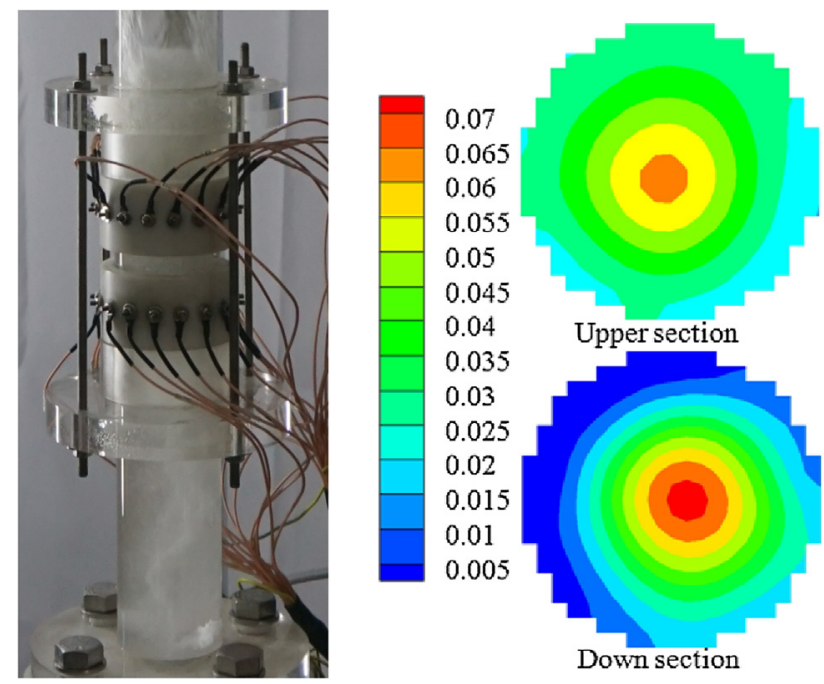

Fig. 19. Local void fraction in the lower part of the GLCC under $V_{s g}=24 \mathrm{~m} / \mathrm{s}$ and $V_{s l}=0.385 \mathrm{~m} / \mathrm{s}$.

the centrifugal intensity in the lower part of the GLCC is weak. It is difficult for the bubbles to migrate to the axis completely. The moisture content of the gas core is high. As can be seen, the average gas holdup in the lower section is less than that in the upper section, which proves the existence of the flow reversal for the gas core. In fact, there exists a pressure gradient in the radial direction of the GLCC due to centrifugal forces. The pressure in the pipe center increases with the decrease in the swirl intensity. The axial pressure gradient develops and reverses the flow direction. The gas core moves up to the free interface where they can disengage. Another distinct observation is that the swirling field presents an obvious nonsymmetry for the single inlet structure. The forming gas core twists and rotates. In a severe case, the gas core can beat the wall of the GLCC, which easily induces vibration and causes structural damage.

4.2.2. Effect of gas-liquid flow rates on the gas carry-under

The gas fraction at the liquid outlet is defined as:

$F_{g}=\frac{Q_{d l}}{Q_{d l}+Q_{d g}}$

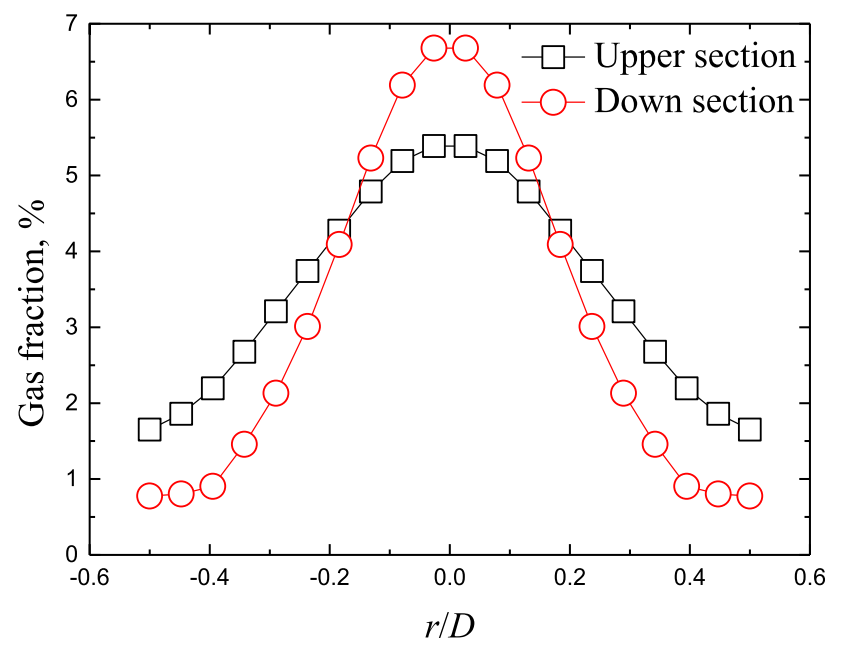

Fig. 20. Radial distribution of the gas fraction. where $Q_{d l}$ is the liquid flow rate at the liquid outlet, and $Q_{d g}$ is the gas flow rate at the liquid outlet.

Fig. 21a displays the variations in the gas fraction at the liquid outlet with the gas superficial velocity. With the increase in the gas superficial velocity, the gas fraction at the liquid outlet gradually decreases and eventually stabilizes. Though denser bubbles are induced for a larger gas superficial velocity, and it can increase the gas core size in the lower part of the GLCC theoretically. However, for a large gas superficial velocity, the liquid occupies a small cross-section area at the inlet nozzle. The tangential velocity of the liquid entering the GLCC increases with a corresponding increase in the swirl intensity. Simultaneously, the high-speed gas can increase the rotational speed of the liquid through the strong shear action at the interface. Both can promote the convergence of the bubbles in a shorter distance. The enhancement effect of swirl intensity wins over the effect of denser bubbles, and thus the gas core size decreases gradually as the gas superficial velocity increases, as shown in Fig. 22. It means that bubbles get well separated from the liquid phase, thereby reducing the gas fraction at the liquid outlet.

Fig. 21b illustrates the effect of the liquid superficial velocity on the gas fraction at the liquid outlet. With the increase in the liquid superficial velocity, the gas fraction at the liquid outlet firstly decreases and then increases. As the liquid superficial velocity increases, the swirl intensity began to increase rapidly. The bubbles then migrated to the axis rapidly, and the gas core was formed. The gas-liquid separation performance was enhanced. However, the gas core could not move up to the free interface in time with a large liquid velocity. This led to an increase in the gas fraction at the liquid outlet.

\subsection{Velocity distribution of gas flow}

Fig. 23 shows the velocity vector distribution near the inlet of the GLCC. The flow at the inlet is very disordered, resulting in many eddies. The fluid under the inlet centerline circulates from top to bottom and then from bottom to top, forming a circulatory eddy flow, which has a negative impact on the gas-liquid separation performance. As can be seen from the velocity vector distribution above the inlet centerline, the fluid flows in a three-dimensional helical manner. Due to the high gas velocity, partial liquid flows directly from the gas outlet with the high-speed gas, thereby forming a short-circuit flow.

The velocity distribution of gas flow is investigated by numerical simulation. Fig. 24 depicts the tangential velocity distribution in different sections of the GLCC. As can be seen, the tangential velocity along the radius direction can be approximately regarded as the combination of free and forced vortices. Basically, the tangential velocity increases first and then decreases from the wall to the axis. The tangential velocity near the wall is zero because of the no-slip boundary condition on the solid wall. According to the boundary layer theory, the viscous force has a great influence on the flow field in the thin layer near the wall of the GLCC. The thin layer is small relative to the diameter of the GLCC. Consequently, the tangential velocity near the wall decreases rapidly from the maximum to zero. At a distance from the solid wall, the influence of the viscous force is much smaller than that of the inertia force. Thus, the tangential velocity begins to decrease slowly along the radius direction, reaching the minimum near the axis. The kinetic energy is converted into static energy. The tangential velocity presents an asymmetric distribution along the radius for the single inlet structure. The minimum and maximum tangential velocities are present on the side that deviates from the inlet. In addition, the amplitude of tangential velocity gradually decreases along the axis, which indicates that the swirl intensity in the GLCC gradually decreases along the axis. 

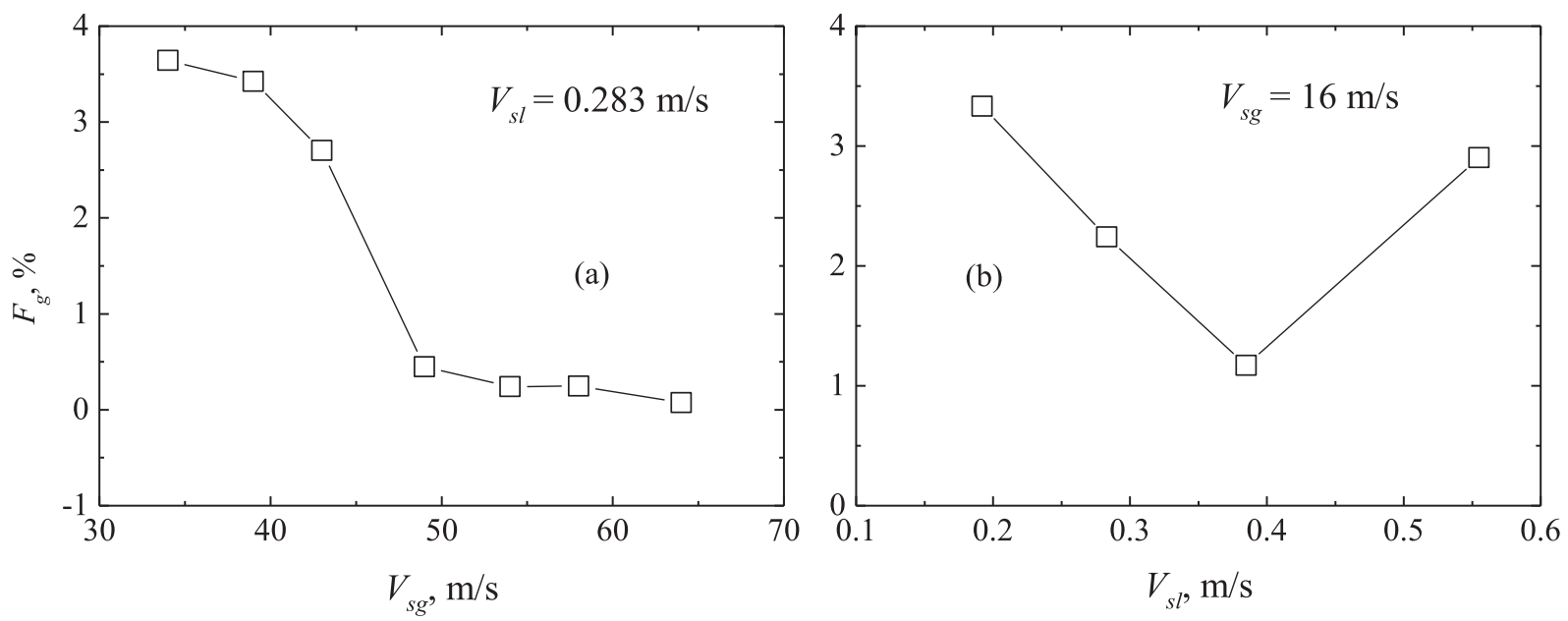

Fig. 21. Effect of the gas (a) and liquid (b) superficial velocity on the gas fraction at the liquid outlet.

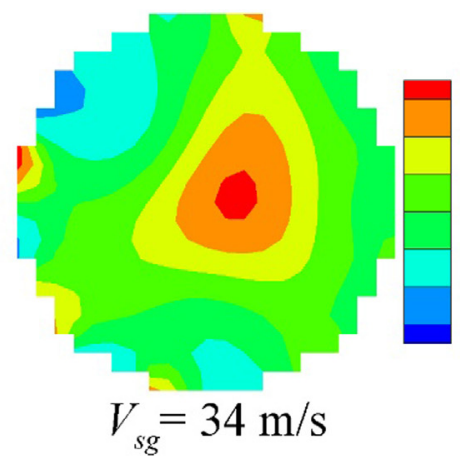

$1.2 \mathrm{E}-02$

$1.0 \mathrm{E}-02$

$8.0 \mathrm{E}-03$

$6.0 \mathrm{E}-03$

$4.0 \mathrm{E}-03$

$2.0 \mathrm{E}-03$

$0.0 \mathrm{E}+00$

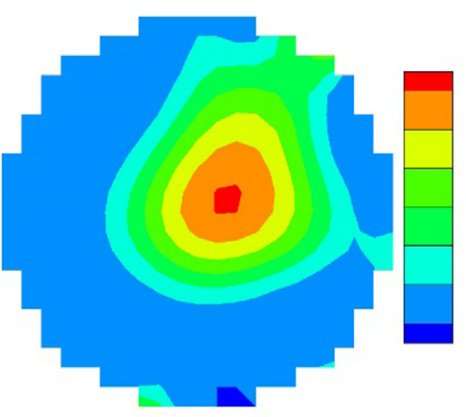

$9.0 \mathrm{E}-03$

7.5E-03

$6.0 \mathrm{E}-03$

$4.5 \mathrm{E}-03$

$3.0 \mathrm{E}-03$

$1.5 \mathrm{E}-03$

$0.0 \mathrm{E}+00$

$V_{s g}=43 \mathrm{~m} / \mathrm{s}$
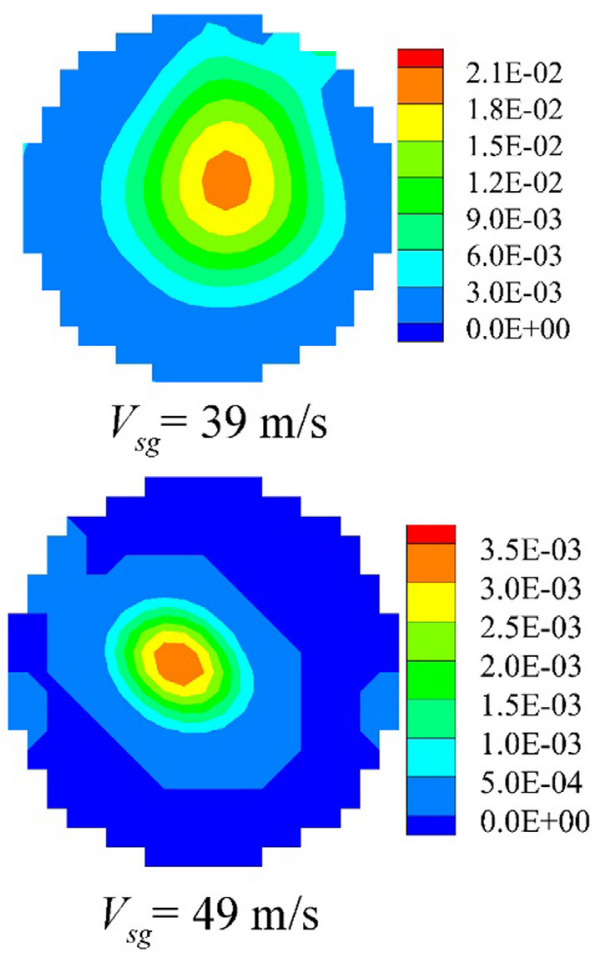

Fig. 22. Cross-sectional void fraction distribution for different gas superficial velocities.

Fig. 25 depicts the axial velocity distribution in different sections of the GLCC. It can be seen from the figure that the axial velocity distribution at the cross-section with a height of $1100 \mathrm{~mm}$ (190 mm above the inlet centerline) is similar to the tangential velocity distribution. Basically, due to the wall effect, the axial velocity is zero on the wall, and first increases and then decreases from the wall to the center. The side that deviates from the inlet presents the minimum axial velocity. With the increase in the axial height, the amplitude of the axial velocity decreases gradually, and the axial velocity distribution along the radial direction stabilizes. The axial velocity gradient is very large in the thin layer of the wall for the large viscous force. At a distance from the solid wall, the axial velocity is not attenuated along the radius.

Fig. 26 depicts the radial velocity distribution in different sections of the GLCC. As can be seen, the radial velocity distributions in different sections are quite different, which indicates that the fluid movement in the radial direction is disordered. The radial velocity increases first and then decreases from the wall to the center of the cyclone. The magnitude of the radial velocity is smaller than those of the tangential velocity and the axial velocity, which is the reason for the measurement difficulty in the experiment (Monredon et al., 1992).

\subsection{Mechanism model}

Based on the above study and the force analysis of a single droplet, the droplet migration model was established. It was assumed that the droplet was released at the axis of the inlet section in the GLCC, as shown in Fig. 27. 


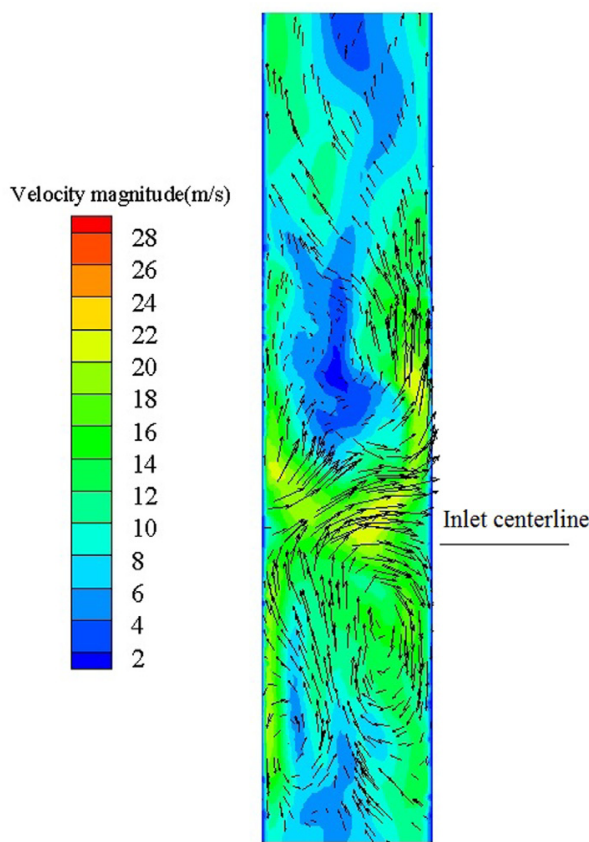

Fig. 23. Distribution of the velocity vector in the GLCC.

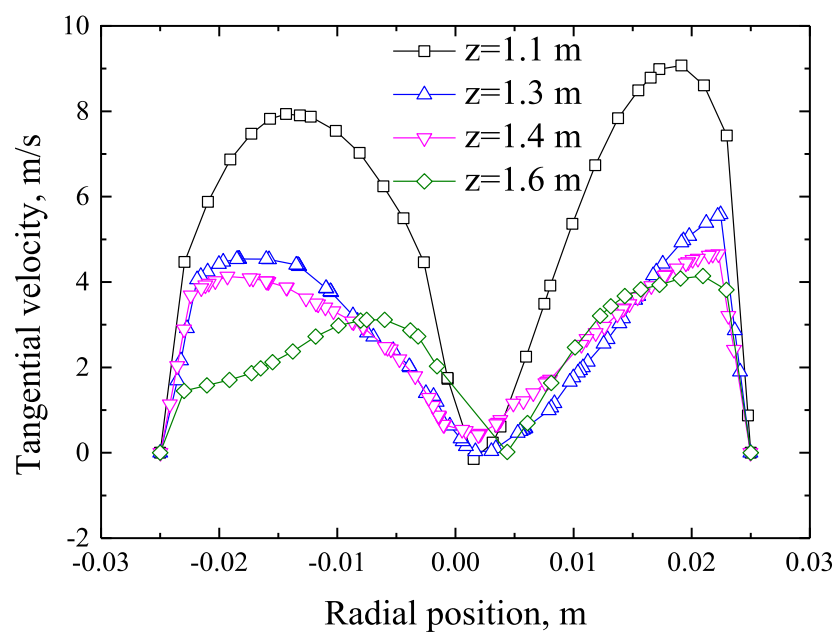

Fig. 24. Radial distribution of the tangential velocity.

The forces acting on the droplet in the continuous gas flow field can be roughly divided into three categories: (1) the forces independent of relative motion, including gravity, gradient force, centrifugal force, and buoyancy force. (2) The forces opposite to the relative motion, including viscous resistance, additional mass force, and basset force. (3) The forces in the same direction as the relative motion, including lift force, Magnus force, and Saffman force. Employing the magnitude analysis for these forces, the main acting force could be determined, namely the viscous resistance, gravity, buoyancy force, gradient force, and centrifugal force.

When relative movement between the droplets and the continuous gas phase occurs, the droplets are subjected to viscous resistance for the viscous effect of the gas. The direction of the viscous resistance is opposite to the actual direction of the droplet motion. The viscous resistance is expressed as:

$\mathrm{F}_{D}=\frac{1}{2} C_{D} \rho_{g} v_{d}^{2} \frac{\pi}{4} d^{2}$

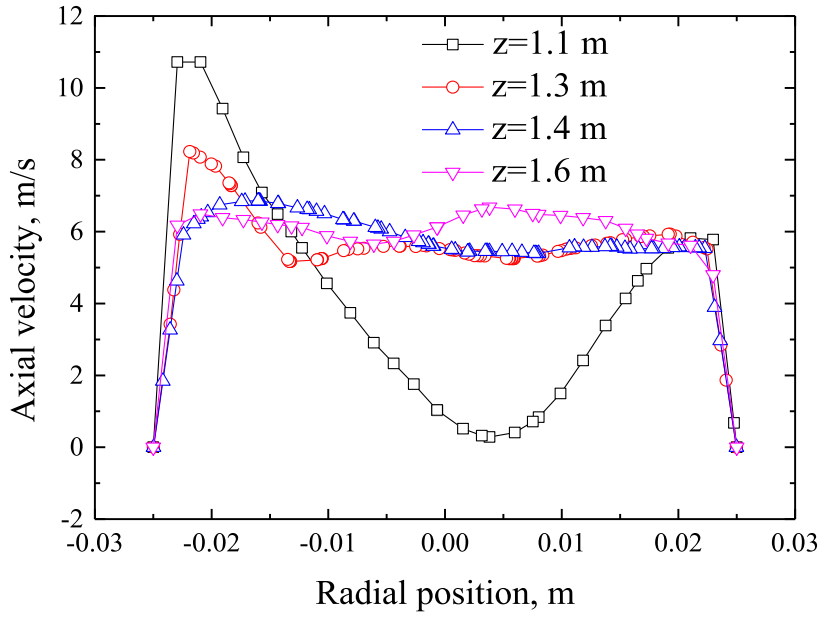

Fig. 25. Radial distribution of the axial velocity.

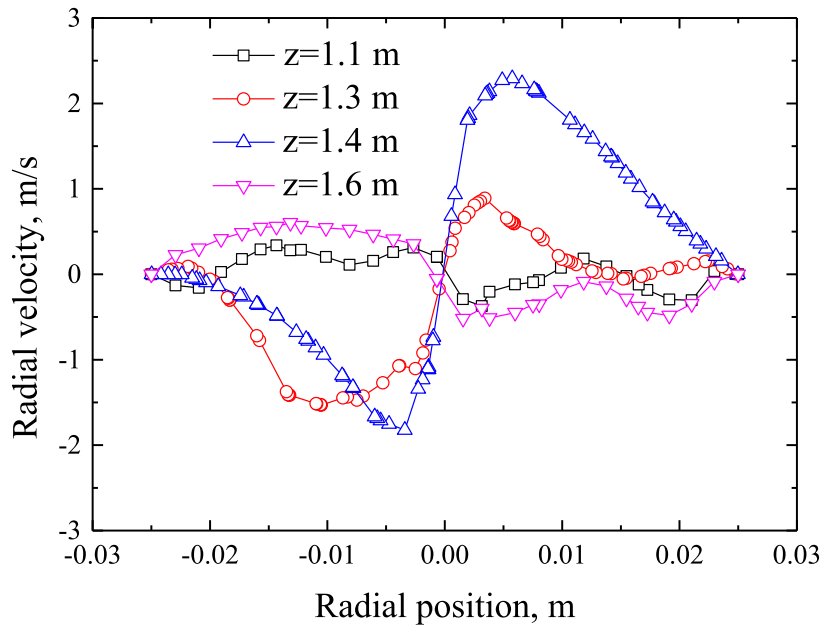

Fig. 26. Radial distribution of the axial velocity.

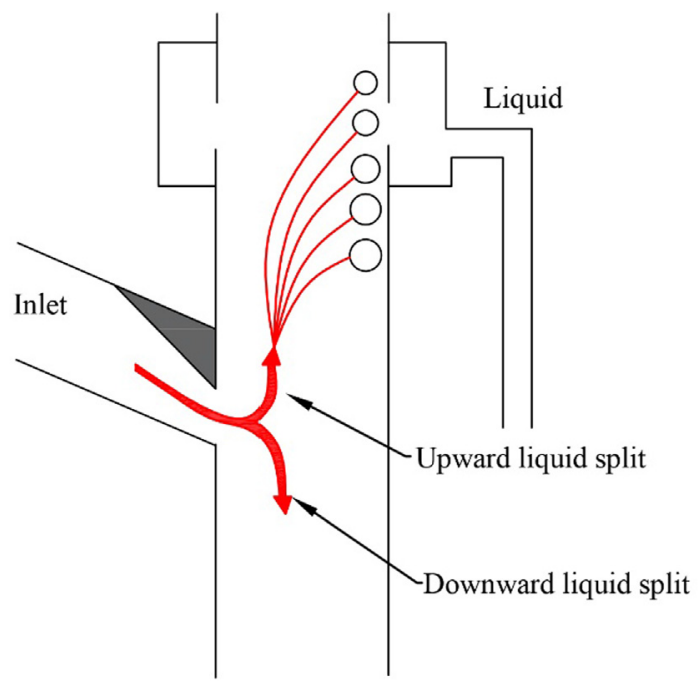

Fig. 27. Schematic of the droplet migration model for the gas-liquid cylindrical cyclone.

where $\rho_{g}$ is the gas phase density; $d$, the droplet diameter; $v_{d}$, the droplet slip velocity; $C_{D}$, the drag coefficient. By comparing different 
drag force models (Yilmaz and Yasar Gundogdu, 2009), the following typical relationship was adopted for the drag coefficient:

$C_{D}=\left\{\begin{array}{cc}24 / \operatorname{Re} & \operatorname{Re}<1 \\ 24\left(1+0.15 \operatorname{Re}^{0.687}\right) / \operatorname{Re} & 1<\operatorname{Re}<1000 \\ 0.44 & \operatorname{Re}>1000\end{array}\right.$

where $\mu_{g}$ is the gas viscosity coefficient. The Reynolds number is given by

$R e=\frac{\rho_{g} v_{d} d}{\mu_{g}}$,

Centrifugal force plays an important role in the gas-liquid separation. The centrifugal force is directed from the axis to the wall. The expression is as follows:

$F_{C}=\frac{\pi}{6} d^{3} \rho_{l} \frac{v_{c t}^{2}}{r}$

where $\rho_{l}$ is gas phase density, and $v_{c t}$ is the tangential velocity of the gas phase.

The resultant force of gravity and buoyancy on the axial direction of the droplets is:

$\mathrm{F}_{\mathrm{cz}}(r)=\frac{\pi}{6} d^{3}\left(\rho_{l}-\rho_{g}\right) g$.

According to the study above, the vortex in the radial direction of the GLCC can be regarded as a combination of the quasi-free vortex and quasi-forced vortex. According to the characteristics of eddy current motion, the pressure near the wall is relatively high and the pressure near the center is relatively low. There is a pressure gradient in the radial direction. The pressure gradient prevents the droplets from moving towards the wall, which is equivalent to the buoyancy in the gravity field. Therefore, it is called the centripetal buoyancy. The centripetal buoyancy points to the axis of the GLCC, and it is calculated as:

$\mathrm{F}_{\mathrm{br}}=\frac{\pi}{6} d^{3} \rho_{g} \frac{v_{c t}^{2}}{r}$

The force balance in the radial direction yields:

$\frac{1}{2} C_{D} \rho_{g} v_{d r} v_{d} \frac{\pi}{4} d^{2}=\frac{\pi}{6} d^{3}\left(\rho_{l}-\rho_{g}\right) \frac{v_{c t}^{2}}{r}$.

Solving Eq. (25) for the slip droplet velocity in the radial direction, yields

$\mathrm{v}_{d r}(r)=\frac{4}{3}\left(\frac{\rho_{l}-\rho_{g}}{\rho_{g}}\right) \frac{v_{c t}^{2}}{r} \frac{d}{C_{D}} \frac{1}{v_{d}}$.

In a similar way, a force balance in the axial direction yields:

$\frac{1}{2} C_{D} \rho_{g} v_{d z} v_{d} \frac{\pi}{4} d^{2}=\frac{\pi}{6} d^{3}\left(\rho_{l}-\rho_{g}\right) g$.

Solving for the axial slip velocity gives

$\mathrm{v}_{d z}(r)=\frac{4}{3}\left(\frac{\rho_{l}-\rho_{g}}{\rho_{g}}\right) g \frac{d}{C_{D}} \frac{1}{v_{d}}$.

Eqs. (26) and (28) can be combined and solved for the total slip velocity of the droplet, as follows:

$\mathrm{v}_{d}(r)=\sqrt{\mathrm{v}_{d r}^{2}(r)+\mathrm{v}_{d z}^{2}(r)}$.

Solving for the droplet velocity yields

$\mathrm{v}_{d}(r)=\sqrt{\frac{4}{3}\left(\frac{\rho_{l}-\rho_{g}}{\rho_{g}}\right) \frac{d}{C_{D}}}\left[g^{2}+\left(\frac{v_{c t}^{2}}{r}\right)^{2}\right]^{1 / 4}$.
The attenuation rate of the swirl intensity along the pipe is a key parameter. The local swirl intensity is defined as the ratio of tangential momentum flux to the total momentum flux at a cross-section in the axial direction:

$\Omega=\frac{2 \pi \rho \int_{0}^{R} v_{s g} v_{c t}(r) r d r}{\rho \pi R^{2} v_{s g}^{2}}$

Chang and Dhir (1994) measured the tangential and axial velocities in the swirling flow field using a hot-wire anemometer. Combined with the data of Kitoh (1991), it was found that the swirl intensity was nearly constant for the axial distance within 2 times the pipe diameter. For other regions, the swirl intensity decreased exponentially along the axial direction, and the ratio of the attenuation rate to the local swirl intensity was a function of the dimensionless axial distance, $(z / D)^{-0.3}$. The expression of the local swirl intensity was obtained as

$\Omega=1.48\left(\frac{M_{t}}{M_{T}}\right)^{0.93} \exp \left[-0.113\left(\frac{M_{t}}{M_{T}}\right)^{0.35}\left(\frac{z}{D}\right)^{0.7}\right]$, for $z / D \geqslant 2$,

$\frac{M_{t}}{M_{T}}=\frac{\dot{m}_{t} A_{D}}{\dot{m}_{T} A_{d}}$.

where $\dot{m}_{t}$ is the gas flow rate at the inlet nozzle; $\dot{m}_{T}$, the gas flow rate in the GLCC; $A_{d}$ and $A_{D}$, the cross-section area of the inlet nozzle and the body of the GLCC, respectively; and $D$, the diameter of the GLCC.

According to the study on tangential velocity in Section 4.4, the quasi-forced vortex is dominant, and the area for the quasi-free vortex is short. Thus, the tangential velocity is approximately linear along the radial direction, the same as the assumption of Gomez et al. (1999). The tangential velocity at the axis is zero and the tangential velocity at the wall is the largest, as given by

$\mathrm{v}_{c t}(r)=\frac{r}{R} v_{c t \max }$,

where $v_{c t \max }$ is the maximum tangential velocity in the radial direction.

Substituting Eq. (34) into Eq. (31), the correlation between the local swirl intensity and the maximum tangential velocity can be obtained as

$\Omega=\frac{2}{3} \frac{v_{c t \max }}{v_{s g}}$

Therefore, the tangential velocity distribution is given by

$\mathrm{v}_{c t}(r)=\frac{3 r v_{s g} \Omega}{D}$

Once $v_{d}$ is solved by Eq. (30) for every radial location of the droplet, the radial and axial components of the slip velocity can be calculated using Eqs. (26) and (28). The trajectory of the droplet can be computed using the following integration:

$\Delta z_{d}=\int \frac{v_{s g}-v_{d z}(r)}{v_{d r}(r)} d r$

The minimum droplet size that reaches the AFE $\left(d_{c r i}\right)$ can be calculated by solving Eq. (37) numerically. If the droplet distribution at the inlet is known, the flow rate of the liquid entering the AFE and gas outlet can be calculated to evaluate the separation efficiency of the GLCC. The extracted liquid carry-over ratio is defined as the ratio of the liquid extracted by AFE to the inlet liquid flow rate: 
$F_{e l}=\frac{Q_{u l} F_{v}\left(d_{c r i}\right)}{Q_{l}}$.

where $F_{v}\left(d_{c r i}\right)$ is the cumulative volume fraction of $d_{c r i}$.

As can be seen from Fig. 28, the predicted droplet diameters agree well with the experimental results for the nozzles with $\mathrm{Ne}=4.0$ and $\mathrm{Ne}=5.9$. However, the predicted droplet diameters are smaller than the measured values for the nozzle with $\mathrm{Ne}=10.7$. The main reason is that the necking ratio is too severe for the nozzle with $\mathrm{Ne}=10.7$. A strong swirling flow causes a strong shear action on the upward moving liquid film, thus forming new droplets. As can be seen in Fig. 29, the predicted and measured values for the extracted liquid carry-over ratio show good concordance. It proves that the developed model correctly establishes the relationship between the characteristics of the swirling flow field and the separation process, which can provide the theoretical guidance for the design of the cyclone separator. This contributes to establishing a stable swirling flow field, realizing rapid accumulation of the droplets, and the rapid phase separation of the gasliquid two-phase.

\section{Conclusion}

Experiments and numerical simulations were conducted to investigate the separation characteristics of the GLCC. A comprehensive understanding of the liquid carry-over, gas carry-under and velocity distribution was obtained. The following conclusions can be drawn.
Experimental results showed that, when the liquid level was below the inlet and above the liquid outlet, it had little effect on the liquid carry-over and the droplet size distribution in the upper part of the GLCC. The separator with strong swirl intensity would not necessarily get better separation performance, and the nozzle with $\mathrm{Ne}=5.9$ performed best.

Malvern RTsizer was used to measure the droplet size distributions before and after separation. It was found that the small and medium droplets tended to initially coalesce and then break up as the gas superficial velocity increased, whereas they always tended to coalesce as the liquid superficial velocity increased. The maximum droplet size at the down sample outlet was mainly determined by the upper swirling flow field that depended on inlet gas superficial velocity and necking ratio.

According to the void fraction distribution measured by ERT imaging system, the gas-liquid separation mechanism in the lower part of the GLCC was investigated. ERT measurement results proved the existence of the flow reversal for the gas core. As the gas flow rate increases, the enhancement effect of swirl intensity wins over the effect of denser bubbles, and thus the gas core size decreases gradually.

DPM model combined with the RNG $k-\varepsilon$ turbulence model was applied to study the swirling hydrodynamics. Considering the effect of droplet breakup and coalescence on the gas flow field, the velocity distribution of gas flow was numerically obtained. Combining with the force analysis of the droplets, the droplet migration model was developed, which accurately predicted the separation performance of the GLCC. The model can be applied to

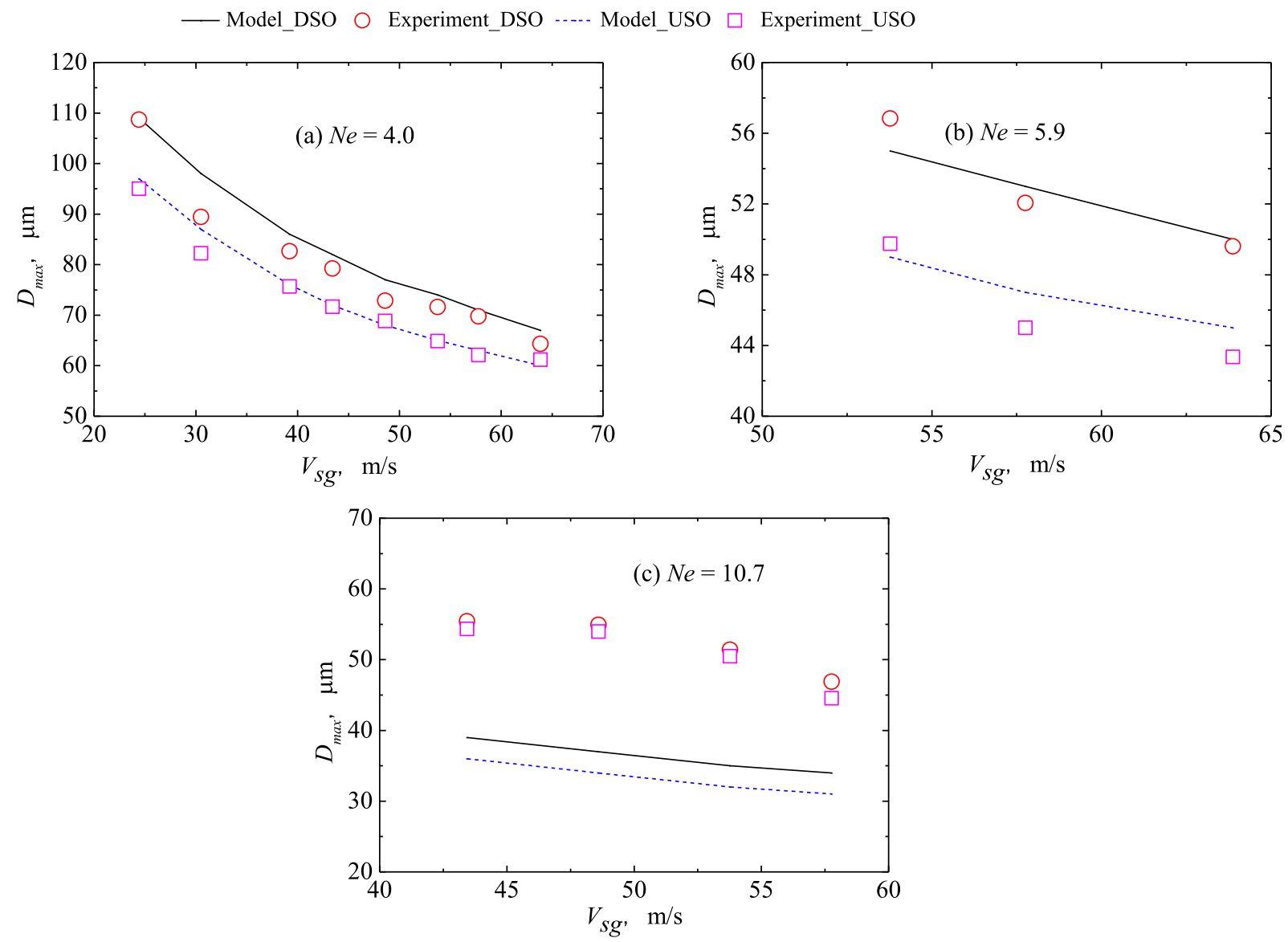

Fig. 28. Comparison between the predicted values and experiment data for the droplet diameter. 

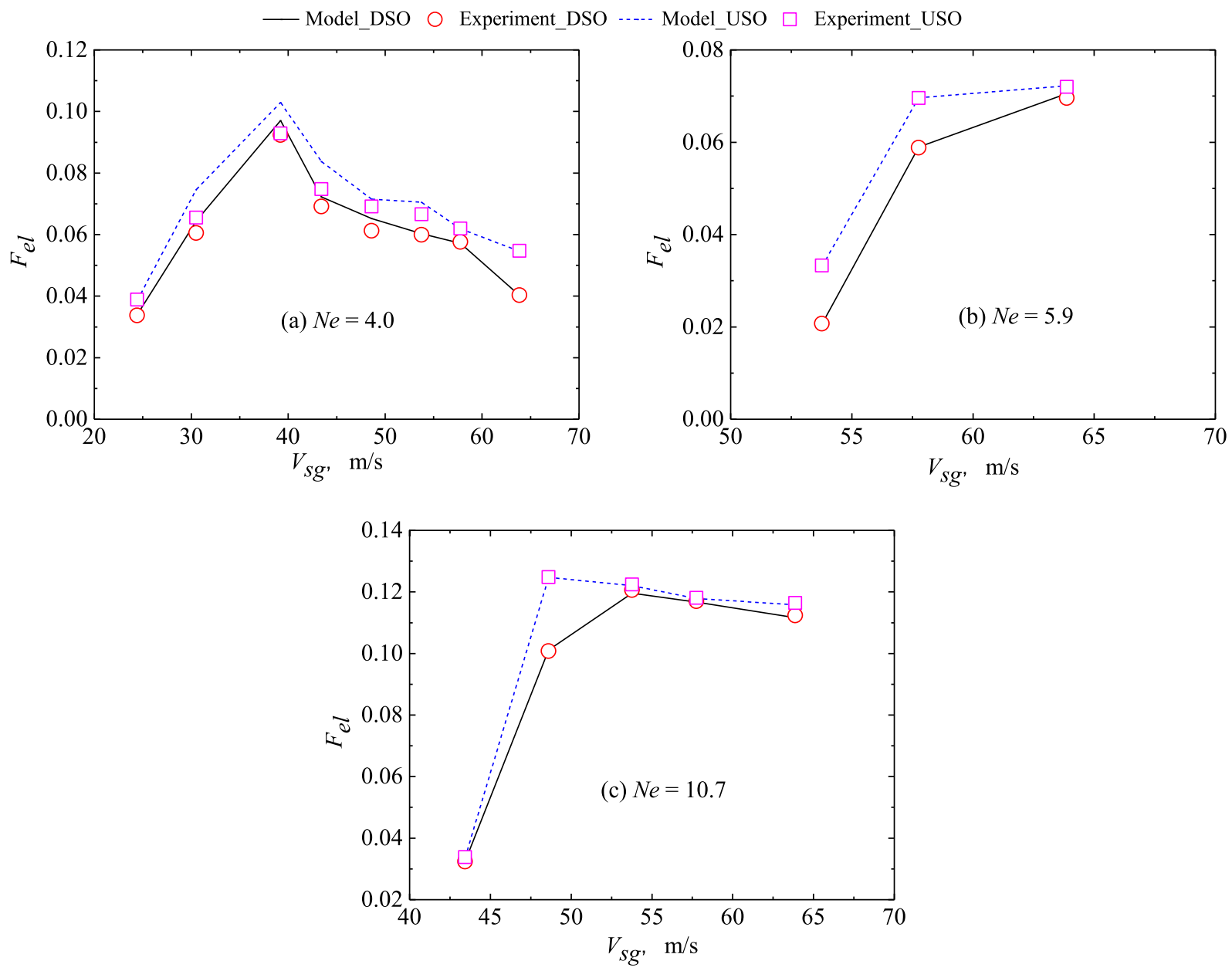

Fig. 29. Comparison between the predicted values and experiment data for the extracted liquid carry-over ratio.

guide the standardized design of the GLCC and realize the rapid accumulation of droplets.

\section{Declaration of Competing Interest}

The authors declare that they have no known competing financial interests or personal relationships that could have appeared to influence the work reported in this paper.

\section{Acknowledgement}

This research was funded by the China Postdoctoral Science Foundation (No. 2019M663242), the Fundamental Research Funds for the Central Universities (No. 19lgpy86) and the Dedicated Fund for Promoting High-Quality Economic Development in Guangdong Province (Marine Economic Development Project) (NO. GDOE2019A43).

\section{References}

Arpandi, I., Joshi, A.R., Shoham, O., Shirazi, S., Kouba, G.E., 1996. Hydrodynamics of two-phase flow in gas-liquid cylindrical cyclone separators. SPE J. 1, 427-436. Bergström, J., Vomhoff, H., 2007. Experimental hydrocyclone flow field studies. Sep. Purif. Technol. 53, 8-20.

Chang, F., Dhir, V.K., 1994. Turbulent flow field in tangentially injected swirl flows in tubes. Int. J. Heat Fluid Fl. 15, 346-356.

Chirinos, W., Gomez, L., Wang, S., Mohan, R., Shoham, O., Kouba, G., 1999. Liquid carry-over in gas-liquid cylindrical cyclone compact separators. SPE Annual Technical Conference and Exhibition. Society of Petroleum Engineers.
Ding, J.W., Li, G.X., Yu, Y.S., 2017. The instability and droplet size distribution of liquid-liquid coaxial swirling spray: an experimental investigation. Exp. Therm. Fluid Sci. 82, 166-173.

Erdal, F.M., Shirazi, S.A., Shoham, O., Kouba, G.E., 1996. CFD simulation of singlephase and two-phase flow in gas-liquid cylindrical cyclone separators. SPE J. 2, 436-446.

Erdal, F.M., Shirazi, S.A., 2004. Local velocity measurements and computational fluid dynamics (CFD) simulations of swirling flow in a cylindrical cyclone separator. J. Energ. Resour. 126, 326-333.

Eshel, G., Levy, G., Mingelgrin, U., Singer, M., 2004. Critical evaluation of the use of laser diffraction for particle-size distribution analysis. Soil. Sci. Soc. Am. J. 68, 736-743.

Gomez, L.E., Mohan, R.S., Shoham, O., Marrelli, J.D., Kouba, G.E., 1999. Aspect ratio modeling and design procedure for GLCC compact separators. J. Energ. ResourAsme. 121, 15-23.

Gupta, A., Kumar, R., 2007. Three-dimensional turbulent swirling flow in a cylinder: experiments and computations. Int. J. Heat Fluid Fl. 28, 249-261.

Han, Q., Zhang, C., Xu, B., Chen, J., 2013. The effect of geometry and operation conditions on the performance of a gas-liquid cylindrical cyclone separator with new structure. AIP Conf. Proc. AIP, 350-361.

Hinze, J., 1955. Fundamentals of the hydrodynamic mechanism of splitting in dispersion processes. AIChE J. 1, 289-295.

Hreiz, R., Gentric, C., Midoux, N., 2011. Numerical investigation of swirling flow in cylindrical cyclones. Chem. Eng. Res. Des. 89, 2521-2539.

Hreiz, R., Gentric, C., Midoux, N., Lainé, R., Fünfschilling, D., 2014. Hydrodynamics and velocity measurements in gas-liquid swirling flows in cylindrical cyclones. Chem. Eng. Res. Des. 92, 2231-2246.

Jia, J., Babatunde, A., Wang, M., 2015. Void fraction measurement of gas-liquid twophase flow from differential pressure. Flow Meas. Instrum. 41, 75-80.

Kitoh, O., 1991. Experimental study of turbulent swirling flow in a straight pipe. J. Fluid Mech. 225, 445-479.

Kouba, G., Shoham, O., 1996. A review of gas-liquid cylindrical cyclone (glcc) technology. In: Production Separation Systems International Conference, Aberdeen, England, April, pp. 23-24.

Kouba, G.E., Shoham, O., Shirazi, S., 1995, Design and performance of gas-liquid cylindrical cyclone separators. In: Proceedings of the BHR Group 7th International Meeting on Multiphase Flow., Cannes, France, pp. 307-327. 
Kusters, K.A., Wijers, J.G., Thoenes, D., 1991. Particle sizing by laser diffraction spectrometry in the anomalous regime. Appl. Optics. 30, 4839-4847.

Lan, Z., Zhu, D., Tian, W., Su, G., Qiu, S., 2014. Experimental study on spray characteristics of pressure-swirl nozzles in pressurizer. Ann. Nucl. Energy. 63, 215-227.

Liu, S., Zhang, D., Yang, L., Xu, J.Y., 2018. Breakup and coalescence regularity of nondilute oil drops in a vane-type swirling flow field. Chem. Eng. Res. Design 129, 35-54.

Mantilla, I., 1998. Bubble Trajectory Analysis in Gas-liquid Cylindrical Cyclone Separators. University of Tulsa.

Meléndez-Ramírez, A.J., Reyes-Gutiérrez, M.A., Rojas-Solorzano, L., 2004. Experimental study of a gas-loquid cylindrical cyclone separator performance. Proceedings of IMECE2004 2004 ASME International Mechanical Engineering Congress, California, USA.

Mohan, R., Wang, S., Shoham, O., Kouba, G., 1998. Design and performance of passive control system for gas-liquid cylindrical cyclone separators. J. Energ. Resour-Asme. 120, 49-55.

Monredon, T.C., Hsieh, K.T., Rajamani, R.K., 1992. Fluid flow model of the hydrocyclone: an investigation of device dimensions. Int. J. Miner Process. 35, 65-83.

Movafaghian, S., Jaua-Marturet, J., Mohan, R.S., Shoham, O., Kouba, G., 2000. The effects of geometry, fluid properties and pressure on the hydrodynamics of gasliquid cylindrical cyclone separators. Int. J. Multiphase Flow. 26, 999-1018.

O'Rourke, P.J., 1981. Collective Drop Effects on Vaporizing Liquid Sprays (Doctoral dissertation). Princeton University.

Patruno, L.E., Marchetti, J., Dorao, C., Svendsen, H., Jakobsen, H., 2010. Droplet size distribution after liquid entrainment in horizontal stratified two-phase threefield dispersed flow. Chem. Eng. Sci. 65, 1407-1414.

Reitz, R., 1987. Modeling atomization processes in high-pressure vaporizing sprays. Atomisation Spray Technol. 3, 309-337.

Reyes-Gutiérrez, M.A., Rojas-Solórzano, L.R., Marín-Moreno, J.C., Meléndez-Ramírez, A.J., Colmenares, J., 2006. Eulerian-eulerian modeling of disperse two-phase flow in a gas-liquid cylindrical cyclone. J. Fluid. Eng. 128, 832-837.
Santolaya, J., García, J., Calvo, E., Cerecedo, L., 2013. Effects of droplet collision phenomena on the development of pressure swirl sprays. Int. J. Multiphase Flow. 56, 160-171.

Sardeshpande, M.V., Gupta, S., Ranade, V.V., 2017. Electrical resistance tomography for gas holdup in a gas-liquid stirred tank reactor. Chem. Eng. Sci. 170, 476-490.

Simmons, M.J., Hanratty, T.J., 2001. Droplet size measurements in horizontal annular gas-liquid flow. Int. J. Multiphase Flow. 27, 861-883.

Singh, B.K., Quiyoom, A., Buwa, V.V., 2017. Dynamics of gas-liquid flow in a cylindrical bubble column: comparison of electrical resistance tomography and voidage probe measurements. Chem. Eng. Sci. 158, 124-139.

Sommerfeld, M., 1998. Analysis of isothermal and evaporating turbulent sprays by phase-Doppler anemometry and numerical calculations. Int. J. Heat Fluid Fl. 19, $173-186$.

Steimes, J., Hendrick, P., 2017. Measurement and prediction of droplet size in annular gas-liquid flows in aero-engine oil systems. Int. J. Multiphase Flow 93 84-91.

Wang, M., Mann, R., Dickin, F., 1999. Electrical resistance tomographic sensing systems for industrial applications. Chem. Eng. Commun. 175, 49-70.

Wang, S., Gomez, L., Mohan, R., Shoham, O., Kouba, G., Marrelli, J., 2010. The stateof-the-art of gas-liquid cylindrical cyclone control technology: from laboratory to field. J. Energ. Resour-Asme. 132, 032701.

Wang, S., 1997. Control System Analysis of Gas-Liquid Cylindrical Cyclone Separators (Doctoral dissertation). University of Tulsa.

Williams, R.A., Ilyas, O.M., Dyakowski, T., Dickin, F.J., Gutierrez, J.A., Wang, M., Rushton, A., 1995. Air core imaging in cyclonic separators: implications for separator design and modelling. Chem. Eng. J. Biochem. Eng. J. 56, 135-141.

Yilmaz, F., Yasar Gundogdu, M., 2009. Analysis of conventional drag and lift models for multiphase CFD modeling of blood flow. Korea-Aust Rheol J. 21, 161-173.

Yue, T., Chen, J., Song, J., Chen, X., Wang, Y., Jia, Z., Xu, R., 2019. Experimental and numerical study of upper swirling liquid film (USLF) among gas-liquid cylindrical cyclones (GLCC). Chem. Eng. J. 358, 806-820.

Zhang, J., Xu, J.Y., 2016. Rheological behaviour of oil and water emulsions and their flow characterization in horizontal pipes. Can. J. Chem. Eng. 94, 324-331. 